\title{
Borehole Data Package for the 216-S-10 Pond and Ditch Well 299-W26-13
}

D. G. Horton

B. A. Williams

C. S. Cearlock ${ }^{(2)}$

May 2000

Prepared for

the U.S. Department of Energy

under Contract DE-AC06-76RLO 1830

Pacific Northwest National Laboratory

Richland, Washington 99352

(a) CH2M Hill Hanford, Inc., Richland, Washington. 



\section{DISCLAIMER}

This report was prepared as an account of work sponsored by an agency of the United States Government. Neither the United States Government nor any agency thereof, nor any of their employees, make any warranty, express or implied, or assumes any legal liability or responsibility for the accuracy, completeness, or usefulness of any information, apparatus, product, or process disclosed, or represents that its use would not infringe privately owned rights. Reference herein to any specific commercial product, process, or service by trade name, trademark, manufacturer, or otherwise does not necessarily constitute or imply its endorsement, recommendation, or favoring by the United States Government or any agency thereof. The views and opinions of authors expressed herein do not necessarily state or reflect those of the United States Government or any agency thereof. 


\section{DISCLAIMER}

Portions of this document may be illegible in electronic image products. Images are produced from the best available original document. 


\section{Contents}

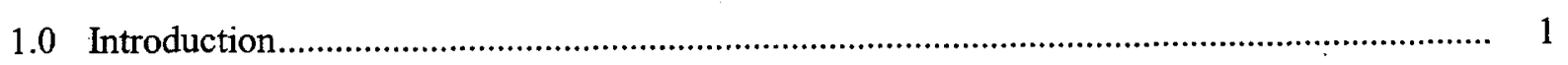

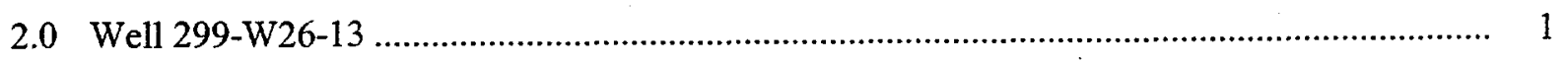

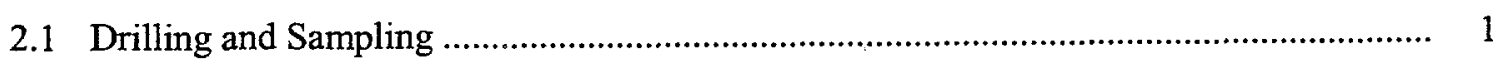

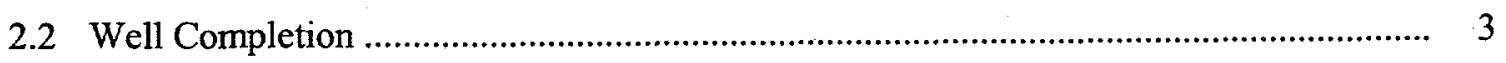

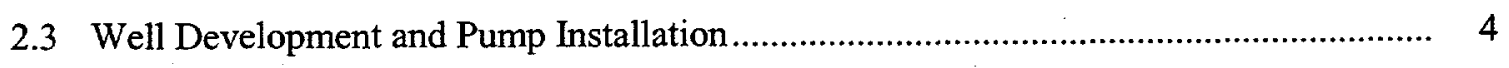

3.0 Vadose Zone Sampling and Analysis ........................................................................... 4

3.1 Sampling ...................................................................................................... 4

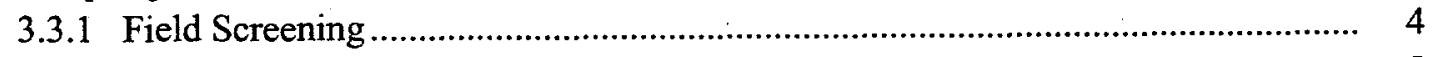

3.1.2 Borehole Sampling ..................................................................................... 5

3.1.3 Geophysical Logging........................................................................................ 5

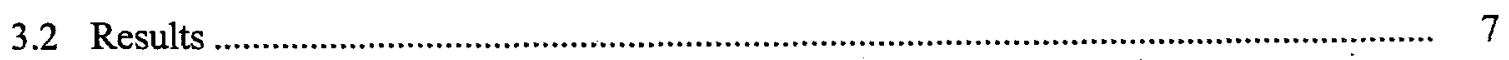

3.2.1 Chemical Analyses .................................................................................. 7

3.2.2 Radiological Analyses ................................................................................. 8

3.2.3 Physical Properties .......................................................................................... 8

3.2.4 Geophysical Logging ................................................................................... 8

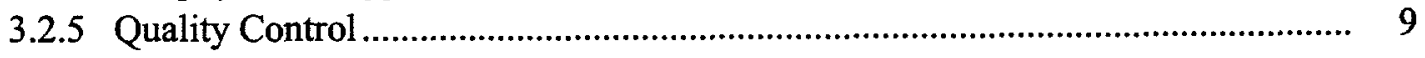

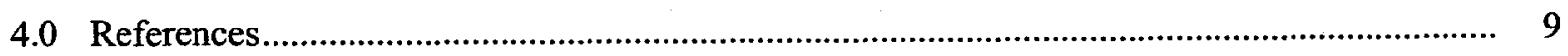

Appendix A - Well Construction and Completion Documentation ............................................. A.1

Appendix B - Sediment Samples Physical and Chemical Properties Data ……............................. B. B.1

Appendix C - Borehole Geophysical Logs............................................................................... C.1 


\section{Figures}

1 Map of 216-S-10 Pond and Ditch and Locations of Wells in the Groundwater

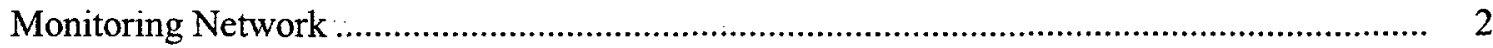

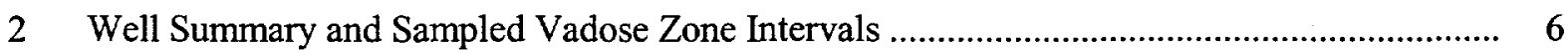

\section{Table}

1 Survey Data for Wells 299-W26-13 at the 216-S-10 Ponds and Ditch .................................. 4 


\subsection{Introduction}

One new Resource Conservation and Recovery Act (RCRA) groundwater monitoring well was installed at the 216-S-10 pond and ditch during November and December 1999 in fulfillment of Tri-Party Agreement (Ecology 1996) milestone M-24-42. The well is 299-W26-13 and is located at the northeast corner to the 216-S-10 pond, southwest of 200 West Area. The well is a new downgradient well in the monitoring network. Figure 1 shows the locations of all wells in the 216-S-10 pond and ditch monitoring network.

The new well was constructed to the specifications and requirements described in Washington Administrative Code (WAC) 173-160 and WAC 173-303, the groundwater monitoring plan for the 216-S-10 pond and ditch (Airhart et al. 1990), and the description of work for well drilling and installation. ' During drilling and construction of well 299-W26-13, sampling and analysis activities were done to support remedial action, closure decisions at treatment, storage and disposal facilities, and to confirm preliminary site conceptual models developed in the 200-CS-1 Work Plan (DOE/RL 1999).

This document compiles information on the drilling and construction, well development, pump installation, and sediment and groundwater testing applicable to well 299-W26-13. Appendix A contains the Well Summary Sheet (as-built diagram), the Well Construction Summary Report, and the geologist's log. Appendix B contains results of field and laboratory determinations of physical and chemical properties of sediment samples. Appendix $\mathrm{C}$ contains borehole geophysical logs. Additional documentation concerning well construction is on file with Bechtel Hanford, Inc., Richland, Washington.

English units are used in this report to describe drilling and well completion activities because that is the system of units used by drillers to measure and report depths and well construction details. Conversion to metric is made by multiplying feet by 0.3048 to obtain meters or multiplying inches by 2.54 to obtain centimeters.

\subsection{Well 299-W26-13}

\subsection{Drilling and Sampling}

Well 299-W26-13 was drilled with a cable tool drill rig and drive barrel between 0 and $52 \mathrm{ft}, 90.7$ and $152 \mathrm{ft}$, and 170 to $240.7 \mathrm{ft}$ below ground surface (bgs). Air rotary was used to drill between 52 and

1 Letter from R. M. Smith, Pacific Northwest National Laboratory, Richland, Washington, to G. C. Henckel, Bechtel Hanford, Inc., dated May 26, 1999, "Description of Work for Drilling of CY 1999 RCRA Groundwater Monitoring Wells." 


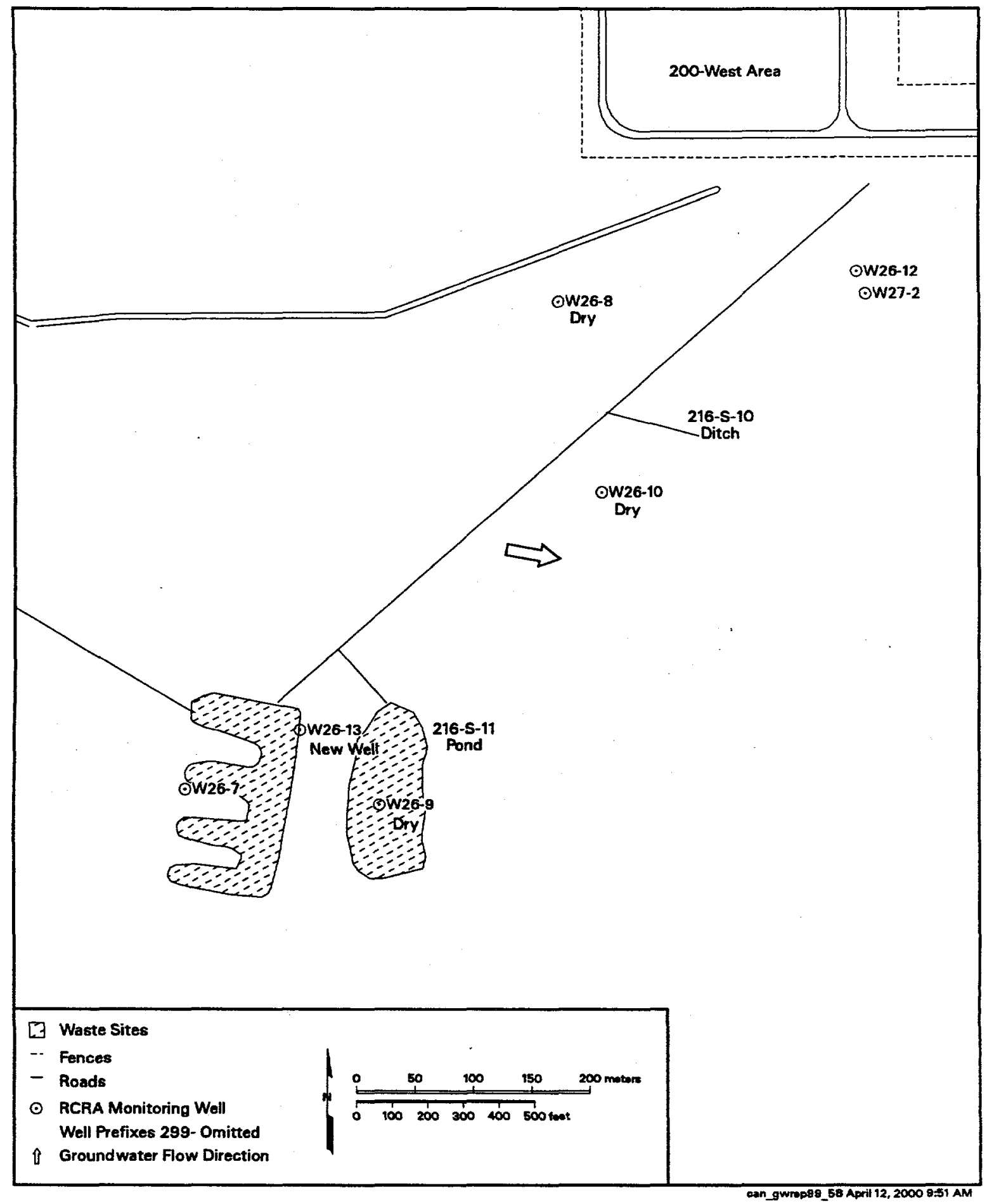

Figure 1. Map of 216-S-10 Pond and Ditch and Locations of Wells in the Groundwater Monitoring Network 
$90.7 \mathrm{ft}$ and between 152 and $170 \mathrm{ft}$ bgs. Temporary $113 / 4$-in.-outside-diameter, carbon steel casing was placed from 0 to $36.3 \mathrm{ft}$ bgs and temporary $85 / 8$-in.-outside-diameter, carbon steel casing was used between 0 and total depth ( $240.7 \mathrm{ft}$ bgs).

Grab samples of sediment for geologic description and archive were collected at approximately $5 \mathrm{ft}$ intervals from $40 \mathrm{ft}$ to total depth. Also, nine 2-ft-long spoon samples were collected for chemical and/or physical properties analyses. Section 3.0 describes the details of sampling and analysis of vadose zone sediments in support of the 200-CS-1 Operable Unit. The results of laboratory analyses are in Appendix B.

Sediments encountered during drilling were predominantly silty to slightly silty sand with minor sand and gravelly sandy silt of the Hanford formation from the surface to about $110 \mathrm{ft}$ bgs; Plio-Pliestocene sandy silt and calcareous sandy silt and silty sand from about 110 to $139 \mathrm{ft}$ bgs; and gravelly sand and sandy gravel of the Ringold Formation from 139 to $240 \mathrm{ft}$ bgs (total depth). A geologist's log is included in Appendix A. The groundwater monitoring plan for the 216-S-10 pond and ditch (Airhart et al. 1990) describes the hydrogeology of the 216-S-10 pond and ditch area.

The borehole and drill cuttings were monitored regularly for organic vapors and radionuclide contaminants. No man-made contamination was noted. The well was geophysically logged using high resolution, spectral gamma-ray and neutron-neutron moisture instrumentation. No man-made radionuclides were detected. The geophysical logs are in Appendix C.

\subsection{Well Completion}

The permanent casing and screen were installed in well 299-W26-13 in December 1999. A 4-in.inner-diameter, stainless steel, continuous wire-wrap (0.01-in. slot) screen was set from 237.3 to $202.2 \mathrm{ft}$ bgs. The permanent casing is 4-in.-inside-diameter, stainless steel from $202.2 \mathrm{ft}$ bgs to $2.9 \mathrm{ft}$ above ground surface. The bottom of the screen has a 4-in. PVC end cap to facilitate later deepening of the well if necessary.

The filter pack is 20 to 40 mesh silica sand from 237.3 to $190.7 \mathrm{ft}$ bgs. The annular seal is bentonite crumbles from 190.7 to $10.3 \mathrm{ft}$ bgs and Portland cement from $10.3 \mathrm{ft}$ to the surface. A $4 \mathrm{ft}$ by $4 \mathrm{ft}$ by 6 in. concrete pad was placed around the well at the surface. A protective casing with locking cap, four protective steel posts, and a brass marker stamped with the well number were set into the concrete. The Well Construction Summary Report and the Well Summary Sheet (as-built) are included in Appendix A.

The vertical and horizontal coordinates of the well were surveyed in March 2000. The horizontal position of the well was determined by Global Positioning System observations referenced to horizontal control stations established by Rogers Surveying, Inc., Richland, Washington. The coordinates are Washington Coordinate System, South Zone, NAD83(91) datum. Vertical datum is NAVD 1988 and is based on existing bench marks established by the U.S. Corps of Engineers. Survey data are included in Table 1. The static water level was $200.05 \mathrm{ft}$ bgs on December 13, 1999. 
Table 1. Survey Data for Well 299-W26-13 at the 216-S-10 Pond and Ditch

\begin{tabular}{|c|c|c|c|c|}
\hline Well Name & $\begin{array}{c}\text { Easting } \\
\text { m (ft) }\end{array}$ & $\begin{array}{c}\text { Northing } \\
\text { m (ft) }\end{array}$ & $\begin{array}{c}\text { Elevation } \\
\text { m (ft) }\end{array}$ & \\
\hline \multirow{3}{*}{$299-W 26-13$} & $\begin{array}{l}566,424.387 \\
(1,858,342.122)\end{array}$ & $\begin{array}{l}133,293.598 \\
(437,313.635)\end{array}$ & & Center of Casing \\
\hline & & & $\begin{array}{l}199.815 \\
(655.559) \\
\end{array}$ & "X" on Casing \\
\hline & $\begin{array}{l}566,424.589 \\
(1,858,342.784)\end{array}$ & $\begin{array}{l}133,293.974 \\
(437,314.869)\end{array}$ & $\begin{array}{l}199.037 \\
(653.007)\end{array}$ & Brass Cap \\
\hline
\end{tabular}

\subsection{Well Development and Pump Installation}

Well 299-W26-13 was developed in December 1999. A temporary, submersible pump was used to remove approximately 9,850 gal of formation water from the well at an initial rate of $18 \mathrm{gal} / \mathrm{min}$ and a final rate of $11 \mathrm{gal} / \mathrm{min}$. The pump intake was at $227.9 \mathrm{ft}$ bgs. The approximate drawdown was $12 \mathrm{ft}$ and the final turbidity was 11.5 NTU.

A dedicated Hydrostar sampling pump was installed in well 299-W26-13 in January 2000. The sampling pump intake is at about $205 \mathrm{ft}$ bgs or about $5 \mathrm{ft}$ below the water table.

\subsection{Vadose Zone Sampling and Analysis}

\subsection{Sampling}

This section describes the collection and analysis of vadose zone samples from well 299-W26-13 in support of the 200-CS-1 Operable Unit.

\subsubsection{Field Screening}

Soil samples were screened in the field prior to sample collection for indications of contamination and to assist with the determination of sample points during drilling. Samples were screened for volatile organic contamination, beta-gamma activity, and alpha activity. Radiological screening was performed by radiation control technicians with an E-600 ratemeter using a SHP380-A/B scintillation probe. Betagamma activities during drilling were at or below background ( 200 to 300 counts per minute) for all sampled intervals. However, a zone of natural occurring radiation was encountered at approximately $197 \mathrm{ft}$ bgs. Radiological field screening indicated a reading of $1,400 \mathrm{cpm}$ of beta-gamma activity. A sample of the material (sample number RCF6872) was sent to the Radiological Counting Facility for analysis. The results indicated that the activity was associated with naturally occurring short-lived 
daughter products of uranium-238 and thorium-232. Volatile organic screening was performed with a photoionization detector. No volatile organics were detected during drilling.

\subsubsection{Borehole Sampling}

Soil samples were collected for analysis of chemical and radiological constituents and for determination of physical properties. The chemical and radiological analyses were done to assess the nature and vertical extent of chemical and radiological contamination. Analyzed constituents included volatile organic and semi-volatile organic compounds, including polychlorinated biphenyls (PCBs), inorganics (metals), total petroleum hydrocarbons, anions, and radionuclides. Physical properties analyses were done to provide input parameters for flow and transport models. Sample collection was guided by the sample schedule in DOE/RL 1999, Appendix B, Tables B.3 to B.5. Samples were analyzed selectively for the following physical properties: field bulk density (using the Bechtel Hanford, Inc. procedure EIP 3.9, Determination of Field Bulk Density Using a Split-spoon Sampler), particle size distribution (by the method ASTM D422), and moisture content (by the method ASTM D2216).

All soil samples were collected with a split-spoon sampler according to BHI-EE-01, Procedure 4.0, "Soil and Sediment Sampling." A total of 10 soil samples were collected from the borehole including 3 quality control samples. The sample locations with respect to ground surface are shown in Figure 2. All analytical results are in Appendix B.

\subsubsection{Geophysical Logging}

Spectral gamma-ray and neutron-neutron geophysical surveys were conducted in borehole 299-W2613. Logging was performed between December 9 and 10, 1999. The spectral gamma-ray and neutronneutron tools were used to determine the concentration of individual man-made and naturally occurring gamma-emitting radionuclides and the volume fraction of moisture (volume of water/volume total) in the soil column. The spectral gamma-ray tool was run from ground surface to a depth of $240.5 \mathrm{ft} \mathrm{bgs.} \mathrm{Data}$ were collected in four log runs within the temporary, 11 - and 8-in.-diameter casings. Two of these log runs were repeat sections to assess repeatability of measurements. Measurements were made at a movestop-acquire mode and at a rate of 100 seconds per $0.5 \mathrm{ft}$.

The moisture tool was run from the bottom of the 11-in.-diameter casing to groundwater ( $36.3 \mathrm{ft}$ to $\sim 200.5 \mathrm{ft}$ ). Data were collected in two log runs that include a repeat section. Moisture data were acquired at a continuous logging speed of $1.0 \mathrm{ft}$ per minute. Ground surface to $36.3 \mathrm{ft}$ was not logged with the moisture tool because it is not calibrated for an 11-in. casing. A detailed report of logging operation is provided in Appendix C. The report describes calibration requirements, data processing, and log plots.

1 Bechtel Hanford, Inc., Richland, Washington. 




$E 0001120.2$

Figure 2. Well Summary and Sampled Vadose Zone Intervals 


\subsection{Results}

Results from the soil sampling and analyses phase of the 299-W26-13 borehole are described in this section. The section includes a discussion of chemical and radiological analyses, physical property testing, geophysical logging data, and quality control.

\subsubsection{Chemical Analyses}

Samples for chemical analyses were sent to an offsite laboratory. All of the analytical results are provided in Tables B.1 through B.7 of Appendix B.

Metal analyses were conducted on all samples and the results are provided in Table B.1. Silver was the only metal not detected in any sample. Arsenic, barium, beryllium, cadmium, total chromium, copper, mercury, nickel, lead, vanadium, and zinc were detected in most of the samples. The concentrations of these metals are generally near or below the Hanford Site background concentrations (DOE/RL 1995). Cadmium and selenium were detected in four samples from 135 to $199 \mathrm{ft}$ bgs with maximum concentrations of $0.5 \mathrm{mg} / \mathrm{kg}$ and $1.9 \mathrm{mg} / \mathrm{kg}$, respectively. Hexavalent chromium was detected only in the 197 to $199 \mathrm{ft}$ bgs interval (directly above the water table) at a concentration of $0.97 \mathrm{mg} / \mathrm{kg}$. Hexavalent chromium is present in the aquifer at this location, and its occurrence in the deepest sample may represent residual contamination in the vadose zone as the aquifer drops.

No polychlorinated biphenyls or diesel range organic compounds were found in any of the samples (Table B.2 and Table B.3, respectively).

Analyses for the anions ammonia, chloride, cyanide, fluoride, nitrate, nitrite, phosphate, sulfate, and sulfide were conducted for the majority of samples and are presented in Table B.4. Cyanide, fluoride, and nitrite were not detected in any sample. The concentrations of the remaining anions were generally less than $5 \mathrm{mg} / \mathrm{kg}$ with a maximum concentration of $12.4 \mathrm{mg} / \mathrm{kg}$ for sulfate.

Analyses for semivolatile organic compounds were conducted on all samples with the exception of the 135 to $137 \mathrm{ft}$ bgs interval. The only contaminant detected was bis (2-ethylhexyl) phthalate with concentrations ranging from 24 to $230 \mu \mathrm{g} / \mathrm{kg}$ (Table B.5). Bis (2-ethylhexly) phthalate is a common laboratory contaminant and was also detected in the quality control blank for the detected maximum value. No other semivolatile organic compounds were detected in any of the samples.

Analyses for volatile organic compounds were conducted on all samples with the exception of the 135 to $137 \mathrm{ft}$ bgs interval. Three target volatile organic contaminants including acetone, 2-butanone, and methylene chloride were detected (Table B.6). Of these, acetone, and methylene chloride were also detected in the quality control blanks and are likely the result of laboratory cross-contamination. 2-butanone was detected once at a concentration of $12 \mu \mathrm{g} / \mathrm{kg}$. No other volatile organic compounds were detected in any of the samples.

The laboratory reported a limited number of volatile and semivolatile tentatively identified compounds. No compounds of significant concern were identified as tentatively identified compounds. 


\subsubsection{Radiological Analyses}

Samples for radiological analyses were sent to an offsite laboratory. All of the results for the radionuclide analyses are provided in Appendix B.

Analyses for both man-made and naturally occurring radionuclides were conducted on all radiological samples. The man-made radionuclides strontium-90, nickel-63, and plutonium-239/240 were each detected once at concentrations of $1.57 \mathrm{pCi} / \mathrm{g}, 2.46 \mathrm{pCi} / \mathrm{g}$, and $0.117 \mathrm{pCi} / \mathrm{g}$, respectively (Table B.7). The nickel-63 and strontium-90 were detected in the duplicate sample at the 50 to $52 \mathrm{ft}$ bgs interval and the plutonium-239/240 was detected in the 99.5 to $101.5 \mathrm{ft}$ bgs interval. No other man-made radionuclides were detected.

The concentration of total uranium was determined for each sample and ranged from 0.46 to $2.14 \mu \mathrm{g} / \mathrm{g}$ (Table B.7). Isotopic analysis for uranium-234, uranium-235, and uranium-238 was conducted for two samples that contained total uranium concentrations above the background level. The concentrations of isotopic uranium compounds detected were less than $0.5 \mathrm{pCi} / \mathrm{g}$.

The concentrations of uranium-238 are generally consistent with those of total uranium (when converted to activity units of $\mathrm{pCi} / \mathrm{g}$ ). The presence of uranium-238 daughter products thorium-230 and radium-226 throughout the vadose zone indicates that the majority of the uranium-238 present is a result of natural rather than nuclear waste disposal processes. Other naturally occurring radionuclides (potassium-40 and thorium-232 plus decay products thorium-228, radium-228, and radium-224) were also detected throughout the vadose zone.

\subsubsection{Physical Properties}

Samples for determination of $\mathrm{pH}$, particle size distribution, and gravimetric moisture content were sent to an offsite laboratory. Bulk density was calculated for three samples based on field measurements. All of the results are provided in Appendix B.

The field $\mathrm{pH}$ measurements ranged from 8.61 to 8.85 (Table B.8). The laboratory pH measurements ranged from 8.1 to 9.1 (Table B.4). The gravimetric moisture content ranged from 2.5 to $11.6 \%$; and bulk density ranged from 1.48 to $2.07 \mathrm{~g} / \mathrm{cm}^{3}$ (Table B.8). Volumetric moisture was also calculated by multiplying the bulk density with the moisture content and dividing by the density of water. Calculated volumetric moisture content ranged from 4.9 to 24.1 (Table B.8). Particle size distribution data are provided in Appendix B.

\subsubsection{Geophysical Logging}

No man-made radionuclides were detected in borehole 299-W26-13. Plots of naturally occurring radionuclides indicate there are several distinct activity changes at depths of about 50,102,133, 195 and $237 \mathrm{ft}$. Major changes correlate to stratigraphic features and with the moisture log data. Potassium- 40 activities typically ranged between 5 and $21 \mathrm{pCi} / \mathrm{g}$; however, a maximum of $34 \mathrm{pCi} / \mathrm{g}$ was detected at a 
depth of $237 \mathrm{ft}$. Uranium-238 and thorium-232 activities ranged between 0.5 to $1.8 \mathrm{pCi} / \mathrm{g}$ and 0.3 to $1.5 \mathrm{pCi} / \mathrm{g}$, respectively. Background values of potassium-40, uranium-238, and thorium 232 are $16.1,1.1$ and $1.32 \mathrm{pCi} / \mathrm{g}$, respectively (DOE/RL 1996).

Volumetric moisture content ranged between 2 and $15 \%$ throughout the logged interval. The maximum value was measured at a depth of $134 \mathrm{ft}$ and correlates to the top of a carbonate-rich zone (caliche). The most obvious features on the moisture plot are the fluctuating values between depths of 90 and $130 \mathrm{ft}$. These depths correlate to a zone of sand with 1 to $2 \mathrm{in}$. thick beds of silt to slightly silty sand. Peaks of high moisture are indicative of the finer grained material within the sand.

\subsubsection{Quality Control Samples}

Quality control samples were collected to evaluate the cross-contamination and laboratory performance. One trip blank and equipment rinsate blank was collected during the field activities.

The trip blank was analyzed only for volatile organics for which no contaminants were detected. The equipment blank was analyzed for volatile and semivolatile organic compounds, anions (except cyanide), metals (excluding hexavalent chromium and mercury), $\mathrm{pH}$, gross alpha and gross beta. One semivolatile compound, di-n-butylphthalate, was detected at an estimated concentration of $1 \mu \mathrm{g} / \mathrm{L}$. Barium and zinc were also in the equipment blank at concentrations of $1.1 \mu \mathrm{g} / \mathrm{L}$. No other contaminants were detected.

Comparison of the analytical results for the sample and the field duplicate were acceptable (relative percent difference values within $35 \%$ ) for most chemical and radiological constituents.

\subsection{References}

Airhart, S. P., J. V. Borghese, and S. Dudziak. 1990. Interim-Status Ground-Water Monitoring Plan for the 216-S-10 Pond and Ditch. WHC-SD-EN-AP-018, Westinghouse Hanford Company, Richland, Washington.

DOE/RL. 1995. Hanford Site Background: Part 1, Soil Background for Inorganics. DOE/RL-92-24, Rev. 3, U.S. Department of Energy, Richland Operations Office, Richland, Washington.

DOE/RL. 1996. Hanford Site Background: Part 2, Soil Background for Radionuclides. DOE/RL-96-12, Rev. 0, U.S. Department of Energy, Richland Operations Office, Richland, Washington.

DOE/RL. 1999. 200-CS-1 Operable Unit RI/FS Work Plan and RCRA TSD Unit Sampling Plan.

DOE/RL-99-44, Draft B, U.S. Department of Energy, Richland, Washington. 
Ecology - Washington State Department of Ecology, U.S. Environmental Protection Agency, and U.S. Department of Energy. 1996. Hanford Federal Facility Agreement and Consent Order. Document No. 89-10, Rev. 4 (The Tri-Party Agreement), Ecology, Olympia, Washington.

RCRA - Resource Conservation and Recovery Act. 1976. Public Law 94-580, as amended, 90 Stat. 2795,42 USC 6901 et seq.

WAC 173-160, Washington Administrative Code. Minimum Standards for Construction and Maintenance of Wells. Olympia, Washington.

WAC 173-303, Washington Administrative Code. Dangerous Waste Regulations. Olympia, Washington. 
Appendix A

Well Construction and Completion Documentation 


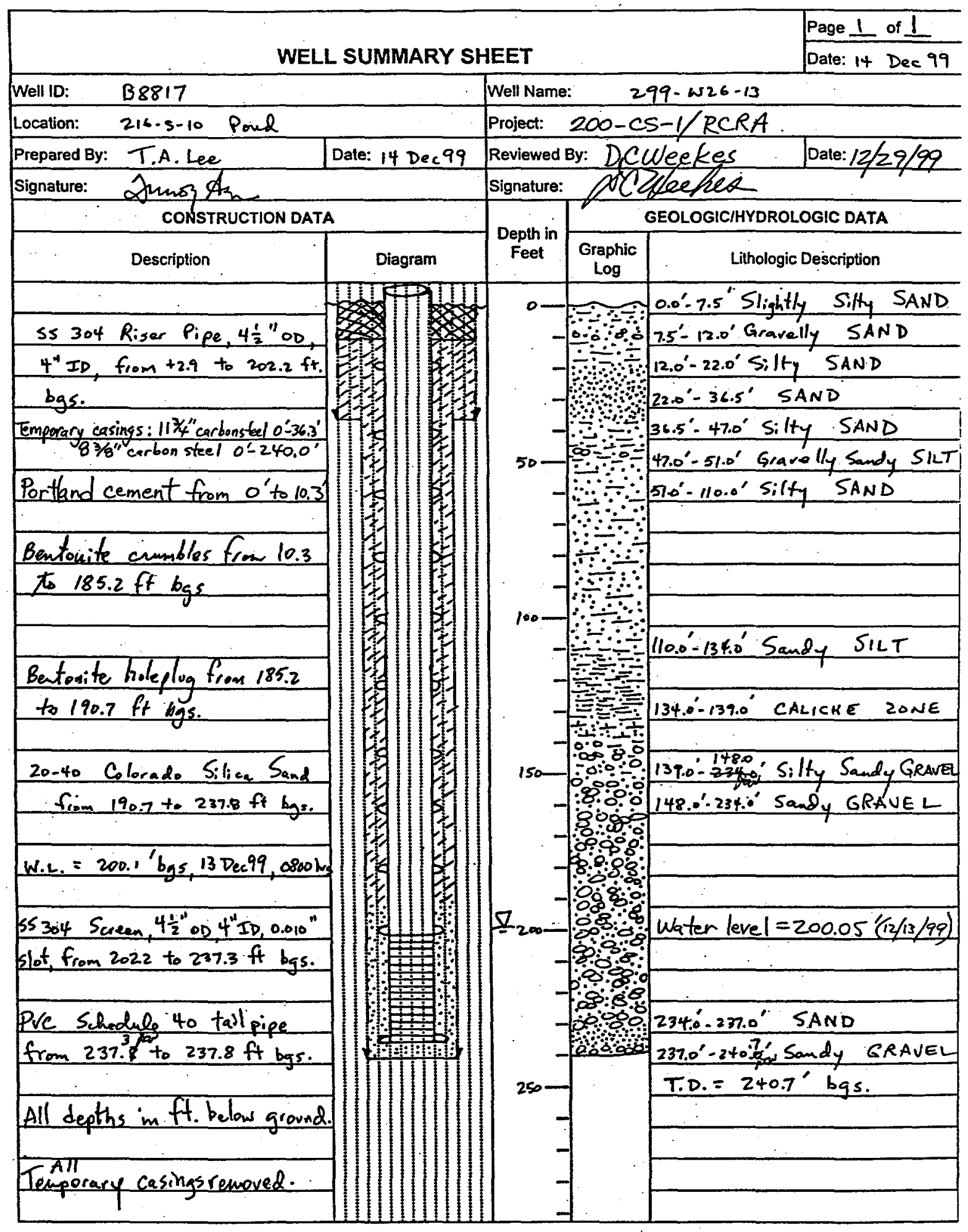

BHI-EE-189 (12/97) 




BHI-EE-181 (12/97) 




BHI-EE-183 (12/97) 


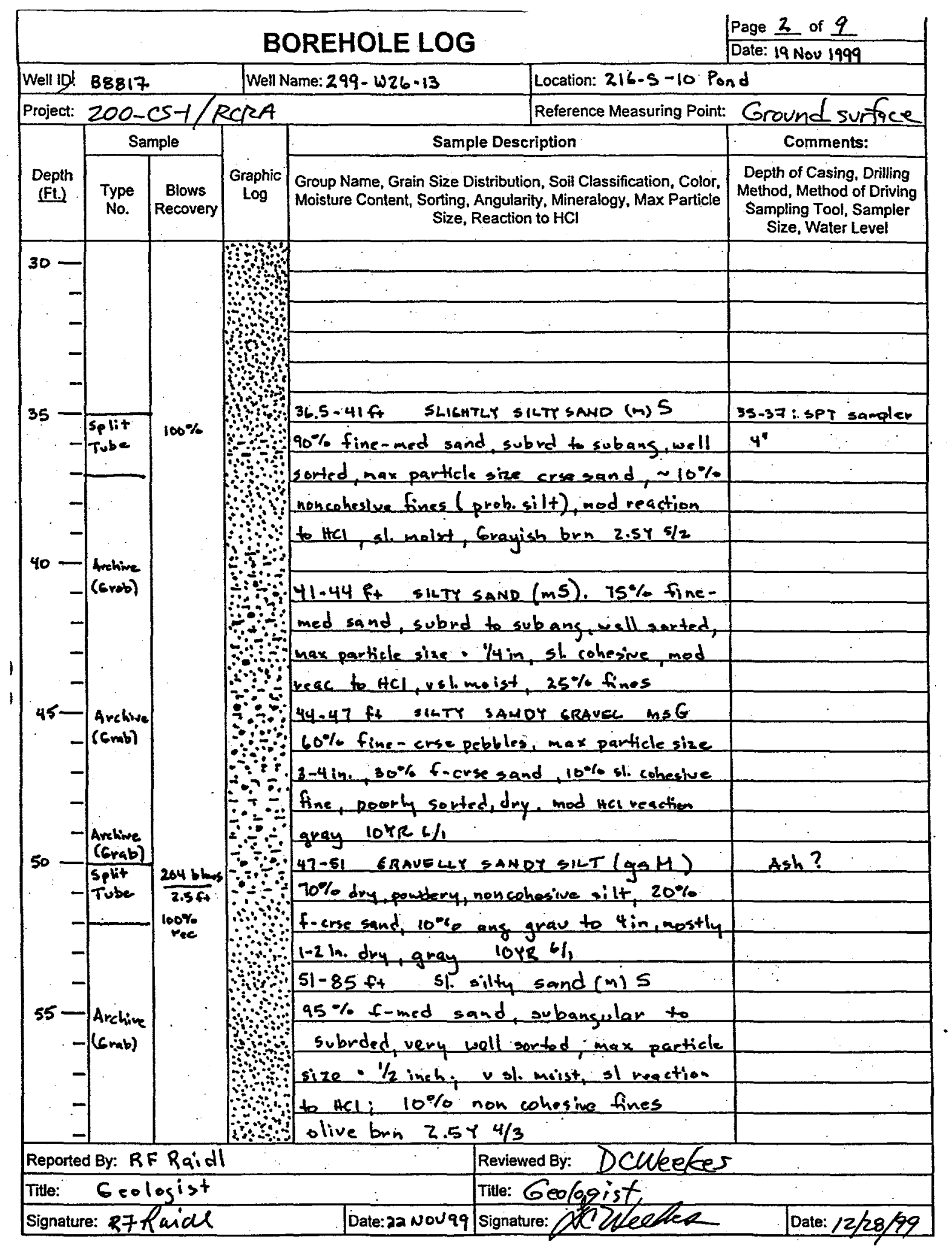

BHI-EE-183 (12/97) 




BHH-EE-183 (12/97) 


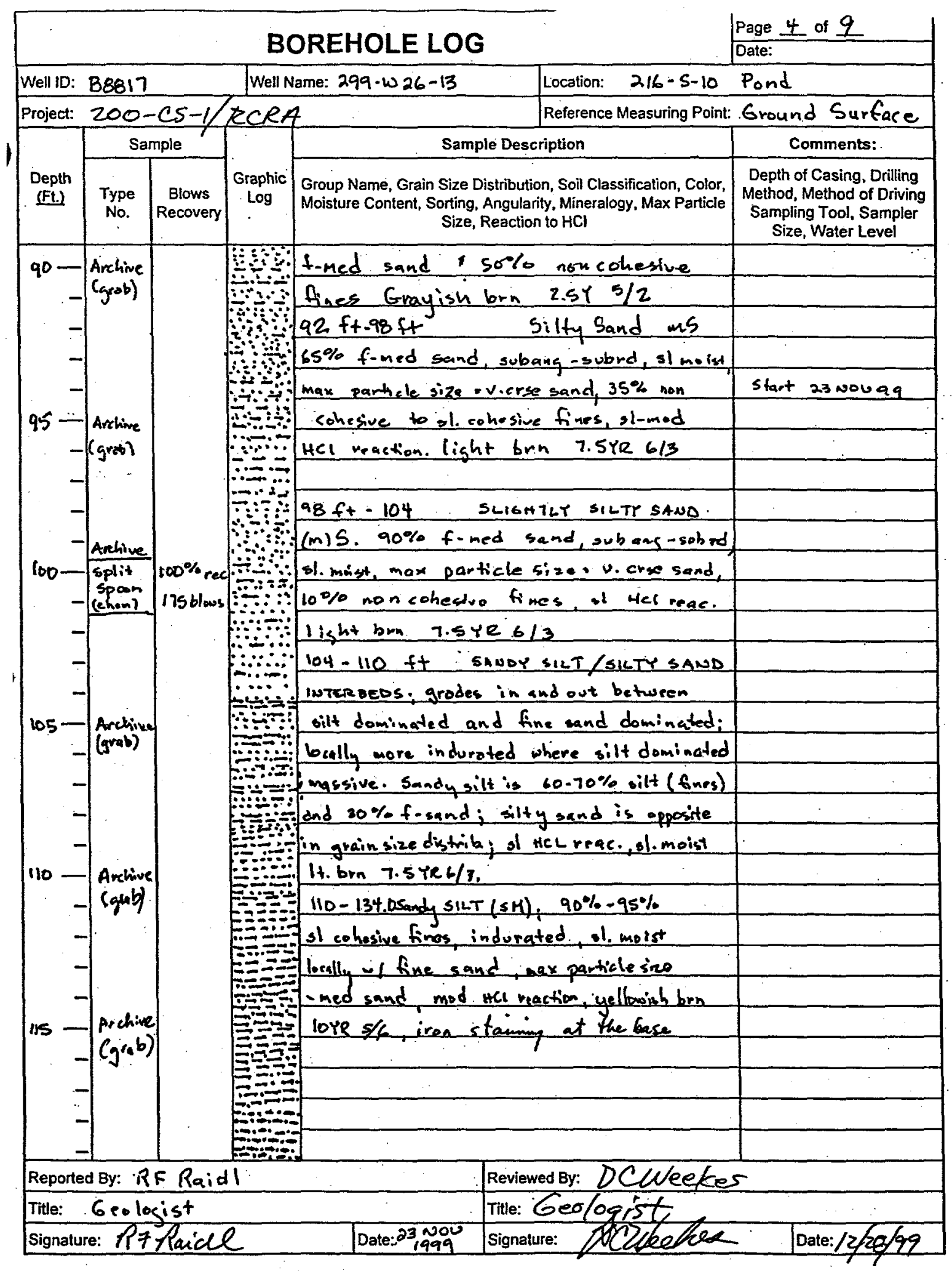

BHI-EE-183 (12/97) 


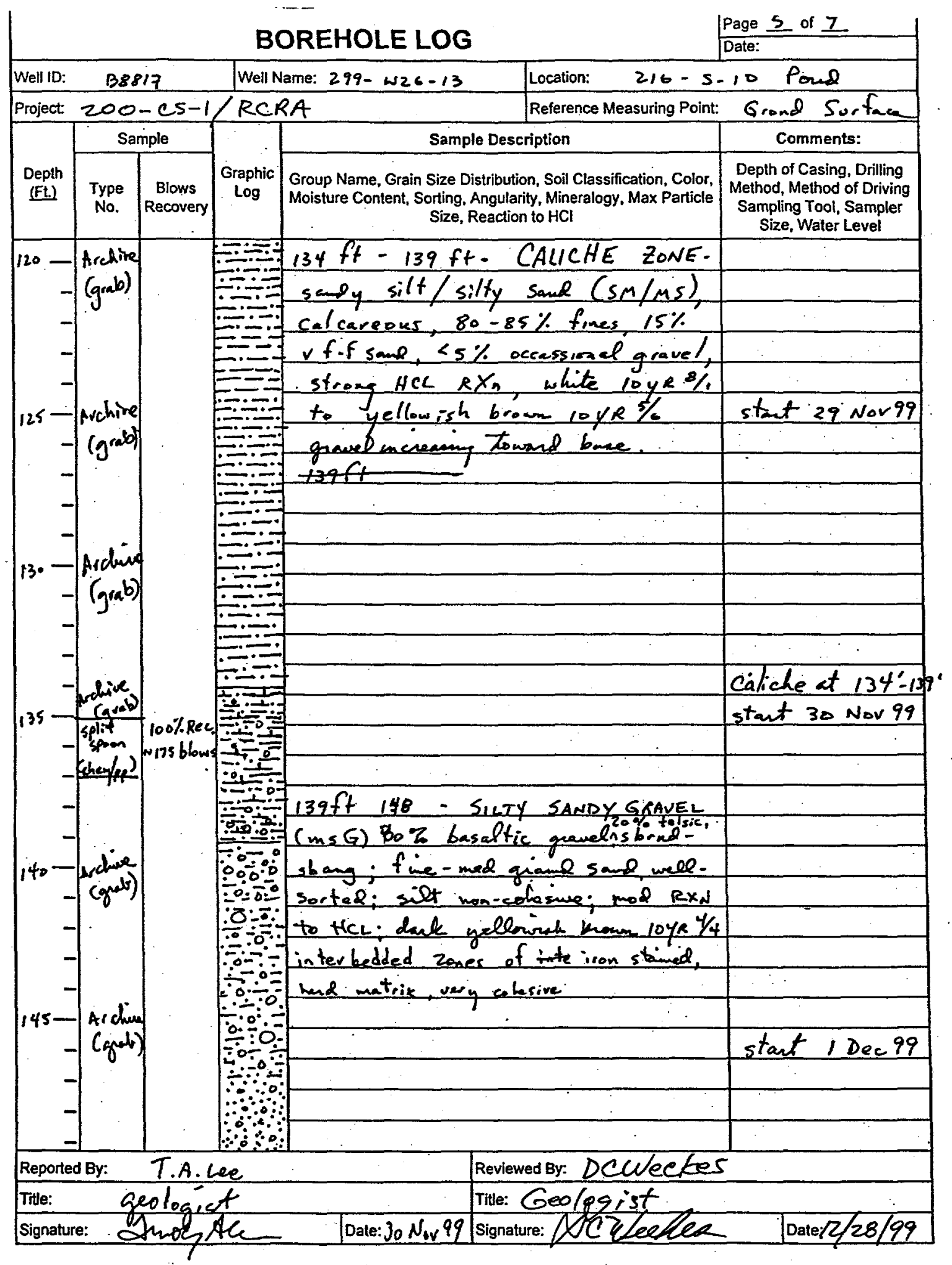

BHH-EE-183 (12/97) 


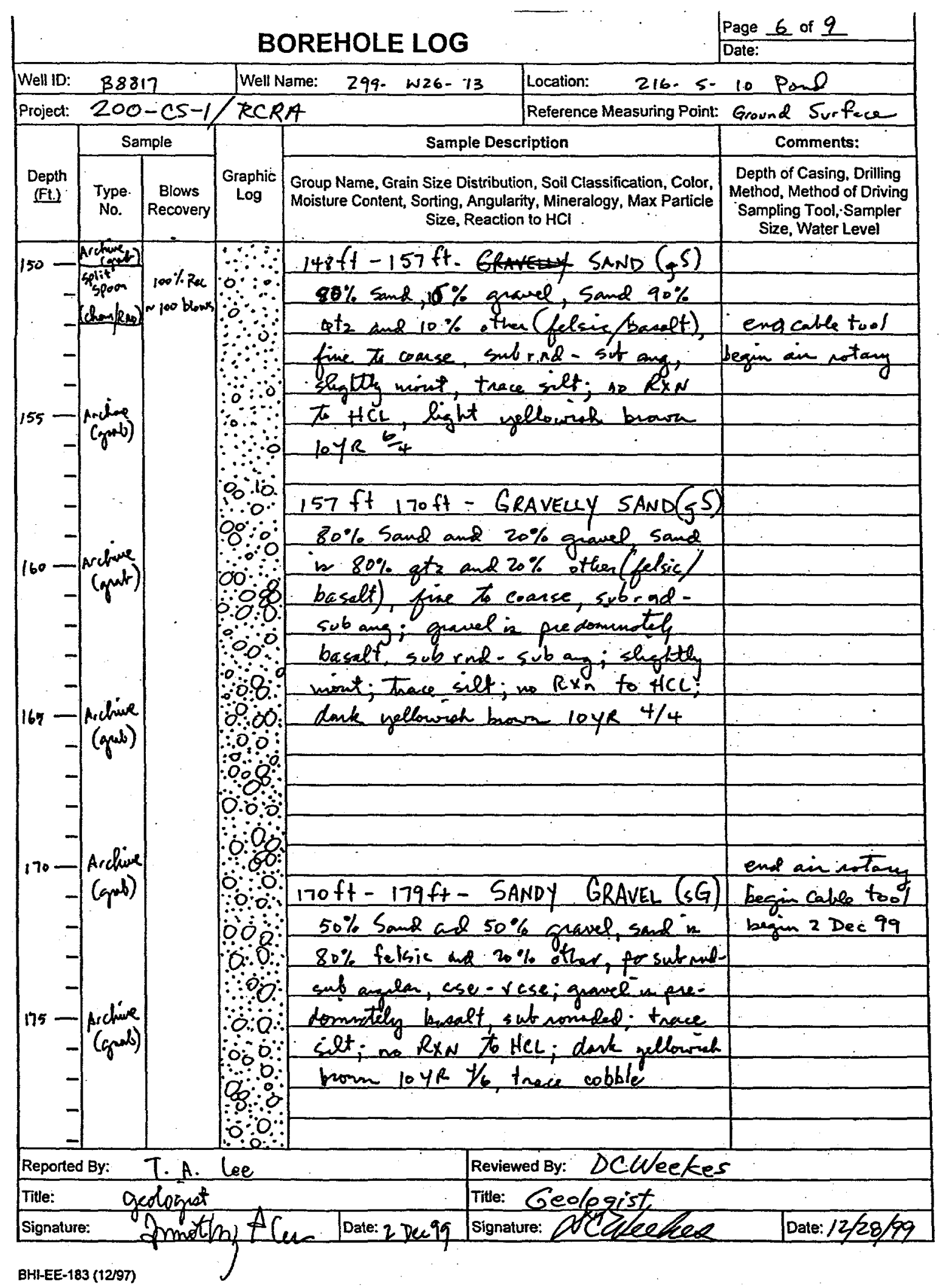




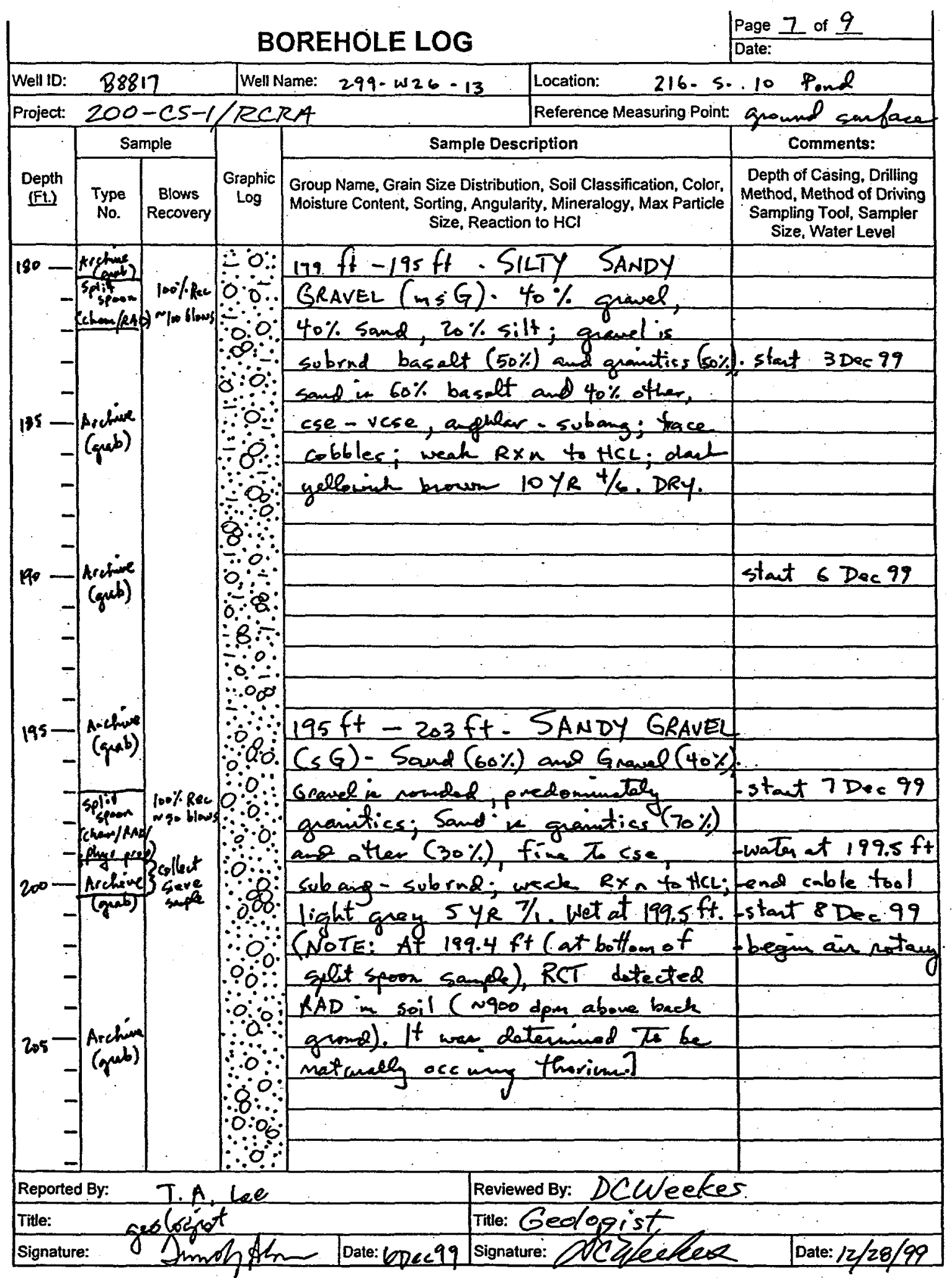

BH-EE-183 (12/97) 


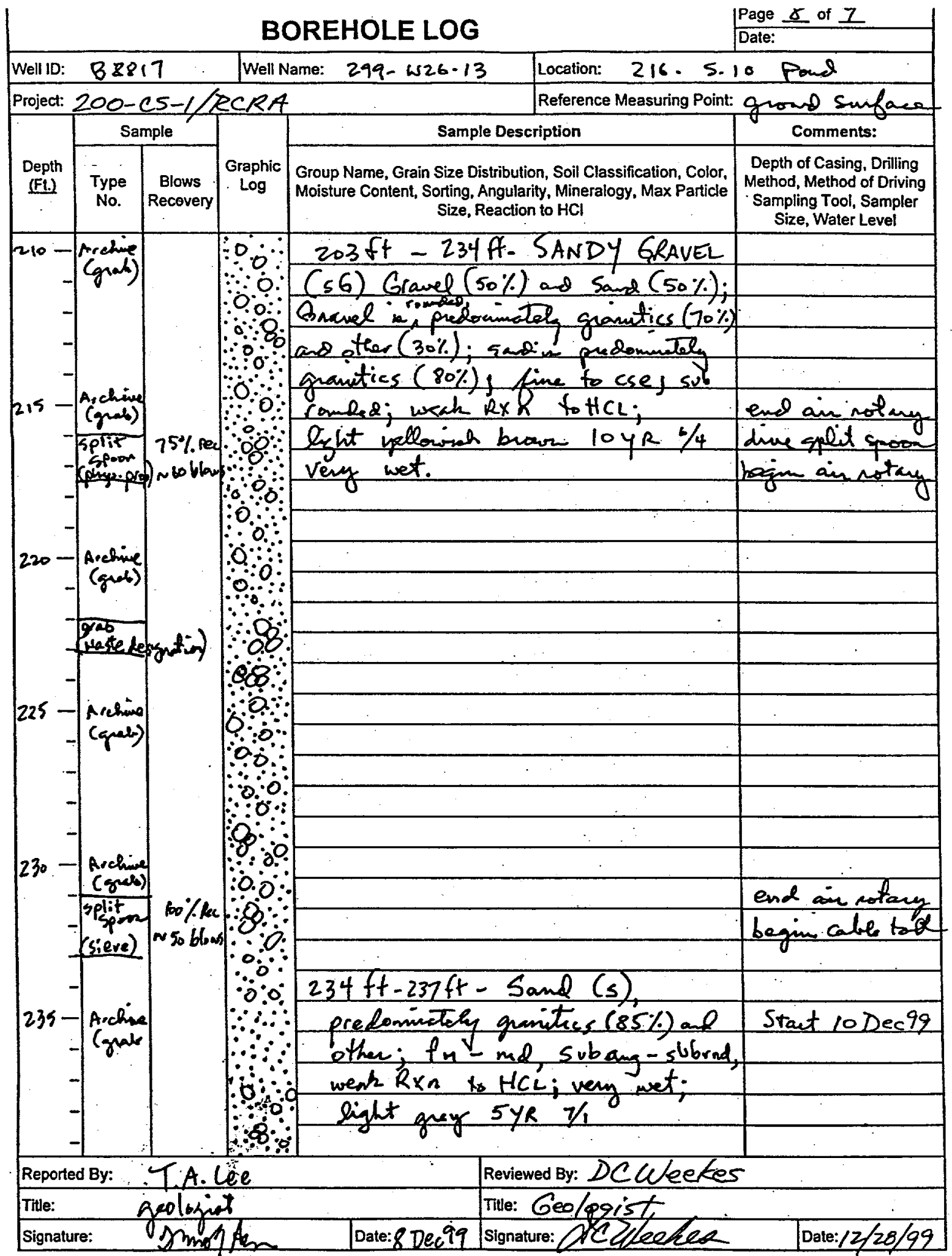

BH-EE-183 (12/97) 


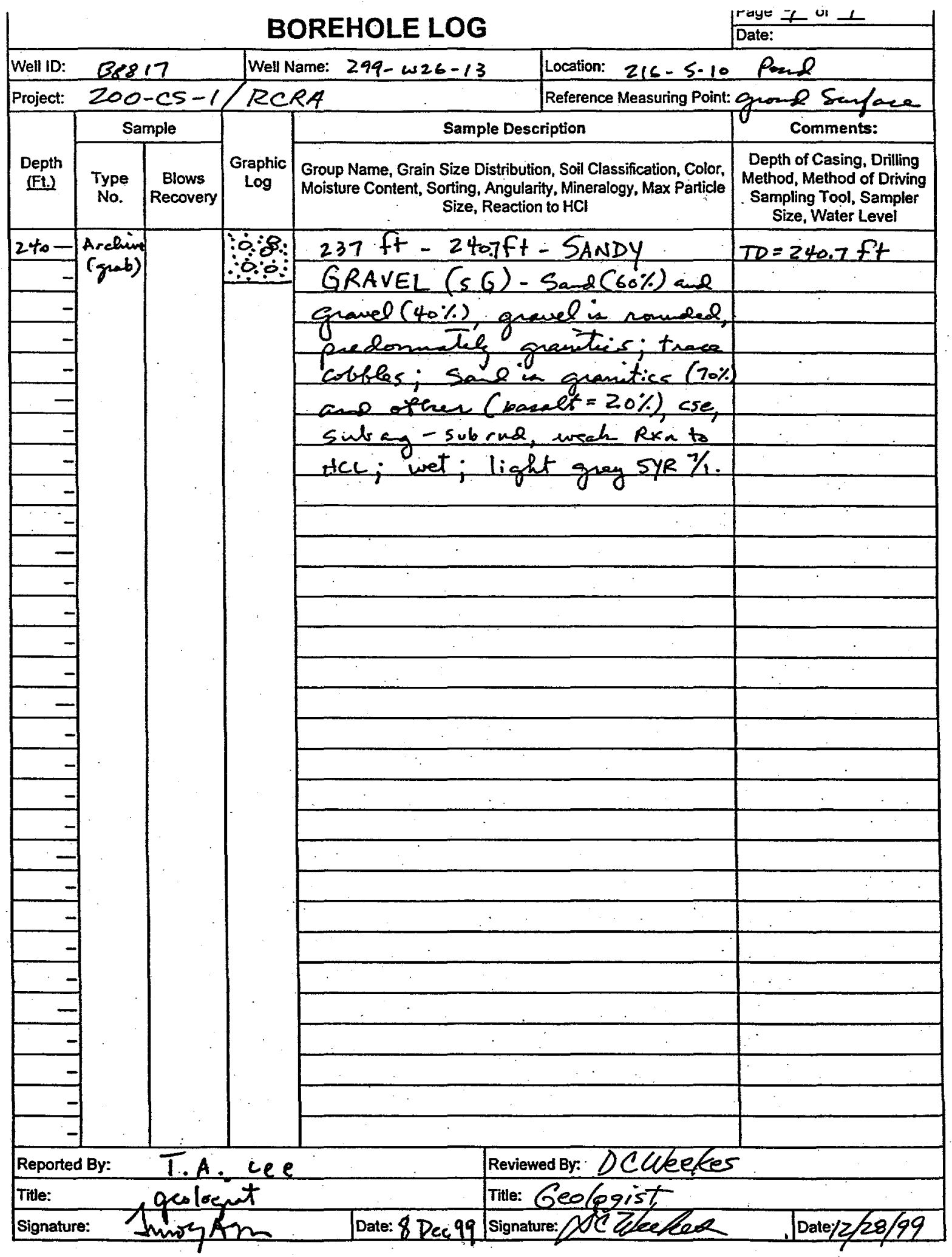

BHI-EE-183 (12/97) 
Appendix B

Sediment Samples Physical and Chemical Properties Data 


\section{Appendix B}

\section{Sediment Samples Physical and Chemical Properties Data}

This appendix includes the results of laboratory testing for chemical and physical properties of sediment samples collected during drilling of well 299-W26-13. The analytical methods and the results are discussed in Section 3.0 of this report. 
Table B.1. Results of Analyses for Metals in Soil Samples from Well 299-W26-13

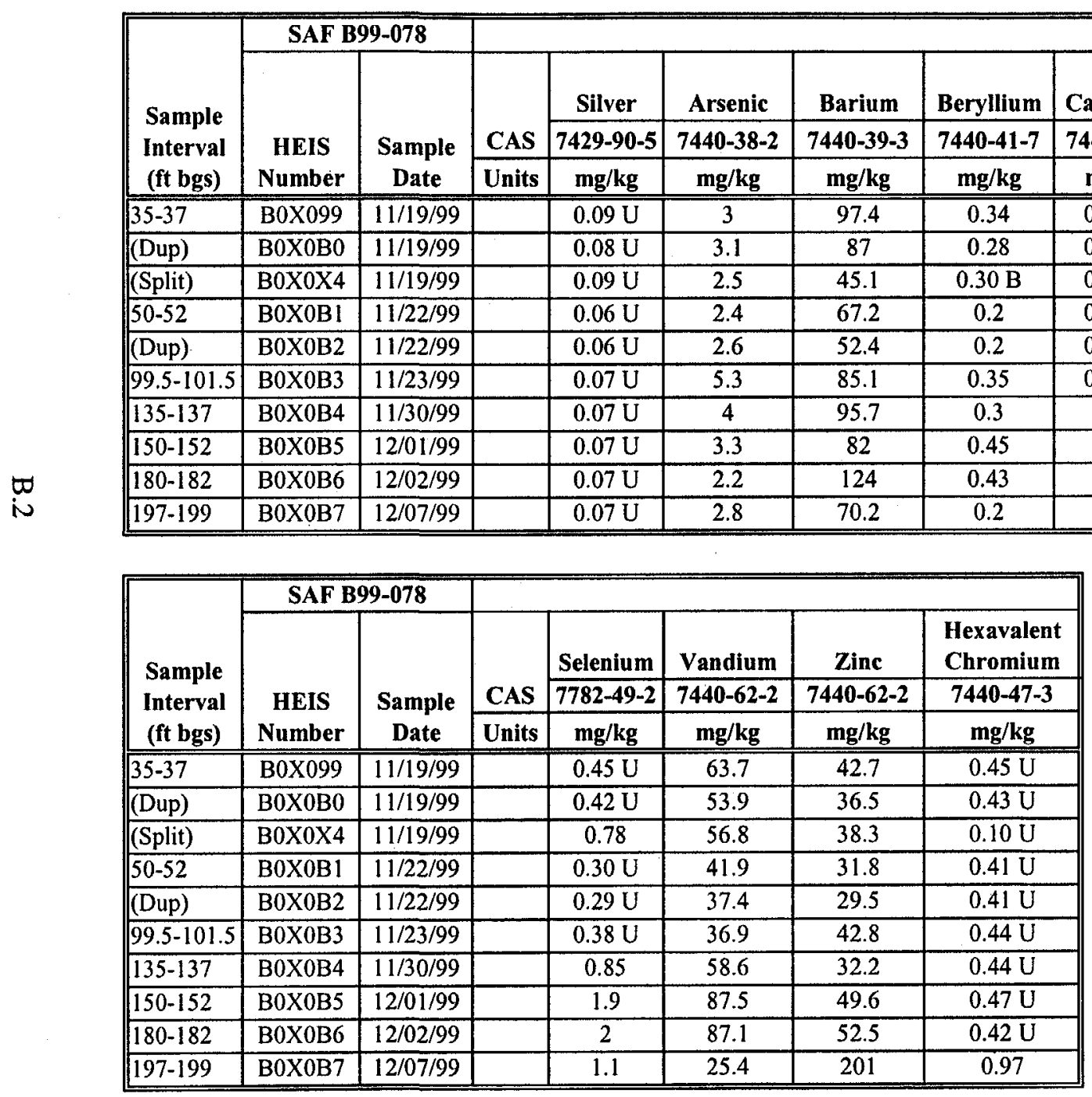

\section{.. = Not analyzed.}

$U=$ Analyzed for but not detected. Value reported is the quantitation limit.

$\mathrm{B}=$ The analyte was detected in the associated $\mathrm{QC}$ blank and in the sample.

$\mathrm{SAF}=$ Sample Authorization Form.

CAS $=$ Chemical Abstract Service registry number.

Dup $=$ Duplicate.

Split $=$ Duplicate sample for quality control purposes. 
Table B.2. Results of Analyses for Polychlorinated Biphenyls in Soil Samples from Well 299-W26-13

\begin{tabular}{|c|c|c|c|c|c|c|c|c|c|c|}
\hline \multirow[b]{3}{*}{$\begin{array}{c}\text { Sample } \\
\text { Interval (ft } \\
\text { bgs) }\end{array}$} & \multicolumn{10}{|l|}{ SAF B99-078 } \\
\hline & \multirow[b]{2}{*}{ HEIS Number } & \multirow[b]{2}{*}{ Sample Date } & \multirow[b]{2}{*}{$\begin{array}{l}\text { CAS } \\
\text { Units } \\
\end{array}$} & $\begin{array}{c}\text { Aroclor } \\
1016 \\
\end{array}$ & \begin{tabular}{|c} 
Aroclor \\
1221 \\
\end{tabular} & \begin{tabular}{|c} 
Aroclor \\
1232 \\
\end{tabular} & $\begin{array}{c}\text { Aroclor } \\
1242\end{array}$ & \begin{tabular}{|c} 
Aroclor \\
1248 \\
\end{tabular} & \begin{tabular}{|c|c} 
Aroclor \\
1254 \\
\end{tabular} & $\begin{array}{r}\text { Aroclo } \\
1260 \\
\end{array}$ \\
\hline & & & & $\begin{array}{l}674-1 \\
\mu \mathrm{g} / \mathrm{kg} \\
\end{array}$ & \begin{tabular}{|c|}
$11104-$ \\
$28-2$ \\
$\mu \mathrm{g} / \mathrm{kg}$
\end{tabular} & $\begin{array}{c}11141- \\
16-5 \\
\mu \mathrm{g} / \mathrm{kg}\end{array}$ & \begin{tabular}{|c|}
$53469-$ \\
$21-9$ \\
$\mu \mathrm{g} / \mathrm{kg}$ \\
\end{tabular} & $\begin{array}{c}12672- \\
29-6 \\
\mu \mathrm{g} / \mathrm{kg}\end{array}$ & $\begin{array}{c}11097- \\
69-1 \\
\mu \mathrm{g} / \mathrm{kg}\end{array}$ & $\begin{array}{c}11096- \\
82-5 \\
\mu \mathrm{g} / \mathrm{kg}\end{array}$ \\
\hline $35-37$ & B0X099 & $111 / 19 / 99$ & & $49 \mathrm{U}$ & $99 \mathrm{U}$ & $49 \mathrm{U}$ & $49 \mathrm{U}$ & $49 \mathrm{U}$ & $49 \mathrm{U}$ & $49 \mathrm{U}$ \\
\hline (Dup) & B0X0B0 & $11 / 19 / 99$ & & $36 \mathrm{U}$ & $72 \mathrm{U}$ & $36 \mathrm{U}$ & $36 \mathrm{U}$ & $36 U$ & $36 \mathrm{U}$ & $36 \mathrm{U}$ \\
\hline (Split) & B0X0X4 & $11 / 19 / 99$ & & -- & -- & -- & -- & -- & -- & -- \\
\hline $50-52$ & $\mathrm{~B} 0 \times 0 \mathrm{~B} 1$ & $11 / 22 / 99$ & & $34 \mathrm{U}$ & $68 \mathrm{U}$ & $34 \mathrm{U}$ & $34 \mathrm{U}$ & $34 \mathrm{U}$ & $34 \mathrm{U}$ & $34 \mathrm{U}$ \\
\hline (Dup) & $\mathrm{B} 0 \mathrm{X0 \textrm {B } 2}$ & $11 / 22 / 99$ & & $34 \mathrm{U}$ & $69 \mathrm{U}$ & $34 \mathrm{U}$ & $34 \mathrm{U}$ & $34 \mathrm{U}$ & $34 \mathrm{U}$ & $34 \mathrm{U}$ \\
\hline $99.5-101.5$ & $\overline{\mathrm{B} 0 \times 0 \mathrm{~B} 3}$ & $11 / 23 / 99$ & & $33 \mathrm{U}$ & $67 \mathrm{U}$ & $33 \mathrm{U}$ & $33 \mathrm{U}$ & $33 \mathrm{U}$ & $33 \mathrm{U}$ & $33 \mathrm{U}$ \\
\hline $135-137$ & $\mathrm{~B} 0 \times 0 \mathrm{~B} 4$ & $11 / 30 / 99$ & & -- & - & -- & -- & -- & - & -- \\
\hline $150-152$ & B0X0B5 & $12 / 01 / 99$ & & $39 \mathrm{U}$ & $78 \mathrm{U}$ & $39 \mathrm{U}$ & $39 \mathrm{U}$ & $39 \mathrm{U}$ & $39 \mathrm{U}$ & $39 \mathrm{U}$ \\
\hline $180-182$ & $\mathrm{~B} 0 \times 0 \mathrm{~B} 6$ & $12 / 02 / 99$ & & $35 \mathrm{U}$ & $71 \mathrm{U}$ & $35 \mathrm{U}$ & $35 \mathrm{U}$ & $35 \mathrm{U}$ & $35 \mathrm{U}$ & $35 \mathrm{U}$ \\
\hline $197-199$ & $\mathrm{~B} 0 \times 0 \mathrm{~B} 7$ & $12 / 07 / 99$ & & $34 \mathrm{U}$ & $68 \mathrm{U}$ & $34 \mathrm{U}$ & $34 \mathrm{U}$ & $34 \mathrm{U}$ & $34 \mathrm{U}$ & $34 \mathrm{U}$ \\
\hline $\begin{array}{l}--=\text { Not ana } \\
\text { U = Analyze } \\
\text { SAF }=\text { Samp } \\
\text { CAS }=\text { Cher } \\
\text { Dup }=\text { Dupl } \\
\text { Split }=\text { Dupl }\end{array}$ & $\begin{array}{l}\text { lyzed. } \\
\text { ed for but not dete } \\
\text { le Authorization } \\
\text { nical Abstract Ser } \\
\text { icate. } \\
\text { licate sample for } \mathrm{q}\end{array}$ & $\begin{array}{l}\text { cted. Value } r \\
\text { Form. } \\
\text { vice registry } r \\
\text { uality control }\end{array}$ & $\begin{array}{l}\text { ber. } \\
\text { poses. }\end{array}$ & . वाना & imit. & & & & & \\
\hline
\end{tabular}

Table B.3. Results of Analyses for Diesel Range Organic Components in Soil Samples from Well 299-W26-13

\begin{tabular}{|c|c|c|c|c|}
\hline \multirow{2}{*}{$\begin{array}{c}\text { Sample } \\
\text { Interval } \\
\text { (ft bgs) }\end{array}$} & \multicolumn{2}{|c|}{ SAF B99-078 } & \multirow[b]{2}{*}{$\begin{array}{l}\text { CAS } \\
\text { Units }\end{array}$} & \multirow{2}{*}{$\begin{array}{c}\text { Diesel Range Organies } \\
\text { DRO } \mathrm{mg} / \mathrm{kg} \\
\end{array}$} \\
\hline & $\begin{array}{c}\text { HEIS } \\
\text { Number }\end{array}$ & $\begin{array}{l}\text { Sample } \\
\text { Date }\end{array}$ & & \\
\hline $35-37$ & B0X099 & $11 / 19 / 99$ & & $4.5 \mathrm{U}$ \\
\hline (Dup) & $\mathrm{B} 0 \times 0 \mathrm{~B} 0$ & $11 / 19 / 99$ & & $4.3 \mathrm{U}$ \\
\hline (Split) & B0X0X4 & $11 / 19 / 99$ & & - \\
\hline $50-52$ & $\mathrm{~B} 0 \mathrm{X} 0 \mathrm{~B} 1$ & $11 / 22 / 99$ & & $4.1 \mathrm{U}$ \\
\hline (Dup) & B0X0B2 & $11 / 22 / 99$ & & $4.1 \mathrm{U}$ \\
\hline $99.5-101.5$ & $\mathrm{~B} 0 \mathrm{X} 0 \mathrm{~B} 3$ & $11 / 23 / 99$ & & $4.4 \mathrm{U}$ \\
\hline $135-137$ & B0X0B4 & $11 / 30 / 99$ & & $\overline{-}$ \\
\hline $150-152$ & B0X0B5 & $12 / 01 / 99$ & & $4.7 \mathrm{U}$ \\
\hline $180-182$ & $\mathrm{~B} 0 \times 0 \mathrm{~B} 6$ & $12 / 02 / 99$ & & $4.2 \mathrm{U}$ \\
\hline $197-199$ & B0X0B7 & $12 / 07 / 99$ & & $4.1 \mathrm{U}$ \\
\hline \multicolumn{5}{|c|}{$\begin{array}{l}--=\text { Not analyzed. } \\
\mathrm{U}=\text { Analyzed for but not detected. Value reported is the quantitation limit. } \\
\text { SAF = Sample Authorization Form. } \\
\text { CAS = Chemical Abstract Service registry number. } \\
\text { Dup = Duplicate. } \\
\text { Split = Duplicate sample for quality control purposes. }\end{array}$} \\
\hline
\end{tabular}


Table B.4. Results of Analyses for Anions in Soil Samples from Well 299-W26-13

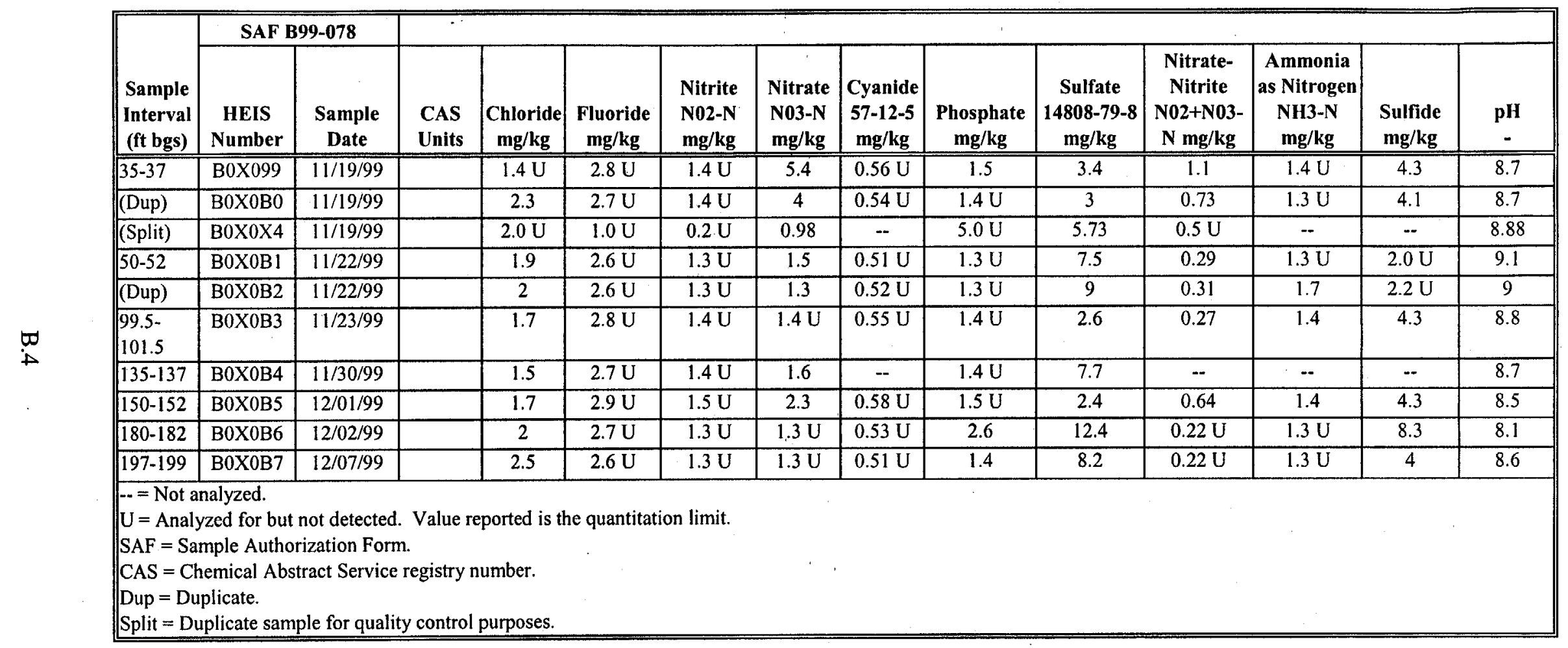


Table B.5. Results of Analyses for Semi-Volatile Organic Compounds in Soil Samples from Well 299-W26-13

\begin{tabular}{|c|c|c|c|c|}
\hline \multirow{2}{*}{$\begin{array}{l}\text { Sample } \\
\text { Interval } \\
\text { (ft bgs) }\end{array}$} & \multicolumn{2}{|c|}{ SAF B99-078 } & \multirow[b]{2}{*}{$\begin{array}{c}\text { CAS } \\
\text { Units }\end{array}$} & \multirow{2}{*}{$\begin{array}{c}\text { Bis } \\
\text { (2-ethylhexyl) } \\
\text { phthalate }\end{array}$} \\
\hline & $\begin{array}{c}\text { HEIS } \\
\text { Number }\end{array}$ & Sample Date & & \\
\hline $35-37$ & B0X099 & $11 / 19 / 99$ & & $370 \mathrm{U}$ \\
\hline (Dup) & $\mathrm{B} 0 \times 0 \mathrm{~B} 0$ & $11 / 19 / 99$ & & $370 \mathrm{U}$ \\
\hline (Split) & B0X0X4 & $11 / 19 / 99$ & & -- \\
\hline $50-52$ & B0X0B1 & $11 / 22 / 99$ & & $24 \mathrm{~J}$ \\
\hline (Dup) & $\mathrm{B} 0 \mathrm{X0B2}$ & $11 / 22 / 99$ & & $27 \mathrm{~J}$ \\
\hline $99.5-101.5$ & $\mathrm{~B} 0 \mathrm{X} 0 \mathrm{~B} 3$ & $11 / 23 / 99$ & & $370 \mathrm{U}$ \\
\hline $135-137$ & $\mathrm{~B} 0 \times 0 \mathrm{~B} 4$ & $11 / 30 / 99$ & & - \\
\hline $150-152$ & B0X0B5 & $12 / 01 / 99$ & & $570 \mathrm{U}$ \\
\hline $180-182$ & B0X0B6 & $12 / 02 / 99$ & & $260 \mathrm{~J}$ \\
\hline $197-199$ & $\mathrm{~B} 0 \mathrm{X0B7}$ & $12 / 07 / 99$ & & $230 \mathrm{JB}$ \\
\hline \multicolumn{5}{|c|}{$\begin{array}{l}--=\text { Not analyzed. } \\
\mathrm{J}=\text { Value reported is an estimate. } \\
\mathrm{B}=\text { The analyte was detected in the associated QC blank and in the sample. } \\
\mathrm{U}=\text { Analyzed for but not detected. Value reported is the quantitation limit. } \\
\text { SAF = Sample Authorization Form. } \\
\text { CAS = Chemical Abstract Service registry number. } \\
\text { Dup = Duplicate. } \\
\text { Split = Duplicate sample for quality control purposes. }\end{array}$} \\
\hline
\end{tabular}

Table B.6. Results of Analyses for Volatile Organic Compounds in Soil Samples from Well 299-W26-13

\begin{tabular}{|c|c|c|c|c|c|c|}
\hline \multirow[b]{2}{*}{$\begin{array}{l}\text { Sample } \\
\text { Interval } \\
\text { (ft bgs) }\end{array}$} & \multicolumn{2}{|c|}{ SAF B99-078 } & \multirow[b]{2}{*}{$\begin{array}{l}\text { CAS } \\
\text { Units }\end{array}$} & \multirow[b]{2}{*}{$\begin{array}{c}\text { Acetone } \\
67-64-1 \\
\mu \mathrm{g} / \mathrm{kg}\end{array}$} & \multirow[b]{2}{*}{$\begin{array}{c}\text { 2-Butanone } \\
78-93-3 \mu \mathrm{g} / \mathrm{kg}\end{array}$} & \multirow[b]{2}{*}{$\begin{array}{c}\text { Methylene } \\
\text { Chloride } 75- \\
09-2 \mu \mathrm{g} / \mathrm{kg}\end{array}$} \\
\hline & $\begin{array}{c}\text { HEIS } \\
\text { Number }\end{array}$ & $\begin{array}{c}\text { Sample } \\
\text { Date }\end{array}$ & & & & \\
\hline $35-37$ & B0X099 & $11 / 19 / 99$ & & $11 \mathrm{U}$ & $11 \mathrm{U}$ & $8 \mathrm{~B}$ \\
\hline (Dup) & $\mathrm{B} 0 \mathrm{X0B0}$ & $11 / 19 / 99$ & & $6 \mathrm{~J}$ & $11 \mathrm{U}$ & $10 \mathrm{~B}$ \\
\hline (Split) & B0X0X4 & $11 / 19 / 99$ & & -- & $\cdots$ & - \\
\hline $50-52$ & $\mathrm{~B} 0 \mathrm{X0B1}$ & $11 / 22 / 99$ & & 30 & $10 \mathrm{U}$ & $6 \mathrm{~B}$ \\
\hline (Dup) & B0X0B2 & $11 / 22 / 99$ & & 22 & $11 \mathrm{U}$ & $7 \mathrm{~B}$ \\
\hline $99.5-101.5$ & B0X0B3 & $11 / 23 / 99$ & & 33 & 12 & $19 \mathrm{~B}$ \\
\hline $135-137$ & B0X0B4 & $11 / 30 / 99$ & & -- & -- & -- \\
\hline $150-152$ & B0X0B5 & $12 / 01 / 99$ & & $22 \mathrm{~B}$ & $12 \mathrm{U}$ & $15 \mathrm{~B}$ \\
\hline $180-182$ & B0X0B6 & $12 / 02 / 99$ & & $6 \mathrm{JB}$ & $11 \mathrm{U}$ & $4 \mathrm{JB}$ \\
\hline $197-199$ & B0X0B7 & $12 / 07 / 99$ & & $5 \mathrm{~J}$ & $11 \mathrm{U}$ & $20 \mathrm{~B}$ \\
\hline \multicolumn{7}{|c|}{$\begin{array}{l}-=\text { Not analyzed. } \\
J=\text { Value reported is an estimate. } \\
\mathrm{B}=\text { The analyte was detected in the associated QC blank and in the sample. } \\
\mathrm{U}=\text { Analyzed for but not detected. Value reported is the quantitation limit. } \\
\mathrm{SAF}=\text { Sample Authorization Form. } \\
\text { CAS = Chemical Abstract Service registry number. } \\
\text { Dup = Duplicate. } \\
\text { Split = Duplicate sample for quality control purposes. }\end{array}$} \\
\hline
\end{tabular}


Table B.7. Results of Analyses for Radionuclides in Soil Samples from Well 299-W26-13

\begin{tabular}{|c|c|c|c|c|c|c|c|c|c|c|c|c|c|c|c|c|}
\hline \multirow[b]{2}{*}{$\begin{array}{l}\text { Sample } \\
\text { Interval } \\
\text { (ft bgs) }\end{array}$} & \multicolumn{2}{|c|}{ SAF B00-006 } & \multirow[b]{2}{*}{$\begin{array}{c}\text { CAS } \\
\text { Units }\end{array}$} & \multirow[b]{2}{*}{$\begin{array}{c}\text { Tritium } \\
10028-17-8 \\
\mathrm{pCi} / \mathrm{g}\end{array}$} & \multirow[b]{2}{*}{\begin{tabular}{|c} 
Technetium \\
9914133-76-7 \\
pCl $/ \mathrm{g}$
\end{tabular}} & \multirow[b]{2}{*}{$\begin{array}{l}\text { Neptunium } \\
237 \text { 13994- } \\
20-2 \mathrm{pCi} / \mathrm{g}\end{array}$} & \multirow[b]{2}{*}{\begin{tabular}{|c|} 
Total \\
Uranium \\
$7440-61-1$ \\
$\mu \mathrm{g} / \mathrm{g}$ \\
\end{tabular}} & \multirow[b]{2}{*}{\begin{tabular}{|l} 
Plutonium \\
$23813981-$ \\
$16-3 \mathrm{pCi} / \mathrm{g}$ \\
\end{tabular}} & \multirow[b]{2}{*}{\begin{tabular}{|c|} 
Plutonium \\
$239 / 240$ \\
PU- \\
$239 / 240$ \\
pCi/g
\end{tabular}} & \multirow[b]{2}{*}{\begin{tabular}{|c|} 
\\
Nickel 63 \\
$13981-37-8$ \\
pCi/g \\
\end{tabular}} & \multirow[b]{2}{*}{$\begin{array}{c}\text { Americium } \\
241 \\
14596-10-2 \\
\mathrm{pCi} / \mathrm{g}\end{array}$} & \multirow[b]{2}{*}{\begin{tabular}{|c|} 
Total \\
Strontium \\
SR-RAD \\
pCi/g \\
\end{tabular}} & \multirow[b]{2}{*}{\begin{tabular}{|c} 
Thorium \\
228 \\
$14274-82-9$ \\
pCi/g \\
\end{tabular}} & \multirow[b]{2}{*}{\begin{tabular}{|c|c|} 
Thorium \\
230 \\
99 \\
$14269-63-7$ \\
$\mathrm{pCi} / \mathrm{g}$
\end{tabular}} & \multirow[b]{2}{*}{$\begin{array}{c}\text { Thorium } \\
232 \\
\text { TH-232 } \\
\text { pCi/g } \\
\end{array}$} & \\
\hline & $\begin{array}{c}\text { HEIS } \\
\text { Number }\end{array}$ & \begin{tabular}{|c|} 
Sample \\
Date
\end{tabular} & & & & & & & & & & & & & & \\
\hline $35-37$ & \begin{tabular}{|l|} 
B0X099 \\
\end{tabular} & \begin{tabular}{|l|l|}
$11 / 19 / 99$ \\
\end{tabular} & & $=$ & -. & - & $0.460 \mathrm{~J}$ & $0.033 \mathrm{U}$ & $0.014 \mathrm{U}$ & - & $0.004 \mathrm{U}$ & $-0.057 \mathrm{U}$ & 0.87 & 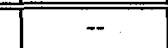 & 0.536 & \\
\hline (Dup) & B0X0B0 & $\mid 11 / 19 / 99$ & & -- & -- & - & 2.14 & $0.036 \mathrm{U}$ & $0.009 \mathrm{U}$ & -- & $0.025 \mathrm{U}$ & $-0.052 \mathrm{U}$ & 0.637 & -- & 0.829 & \\
\hline (Split) & $\begin{array}{ll}\mathrm{B} 0 \mathrm{X} 0 \mathrm{X} 4 \\
\end{array}$ & \begin{tabular}{|l|}
$11 / 19 / 99$ \\
\end{tabular} & & -- & -- & - & $0.614 \mathrm{~J}$ & 0.00226 & $0 \mathrm{U}$ & - & $0.0094 \mathrm{U}$ & $0.0264 \mathrm{U}$ & -- & -- & - & \\
\hline $50-52$ & $\mathrm{~B} 0 \times 0 \mathrm{~B} 1$ & $\mid 11 / 22 / 99$ & & $0.064 \mathrm{U}$ & $-0.062 \mathrm{U}$ & $0.030 \mathrm{U}$ & 1.94 & $0.026 \mathrm{U}$ & $0.015 \mathrm{U}$ & $0.758 \mathrm{U}$ & $0.028 \mathrm{U}$ & $-0.015 \mathrm{U}$ & $0.475 \mathrm{~J}$ & 1.06 & $0.387 \mathrm{~J}$ & \\
\hline (Dup) & $\mathrm{B} 0 \mathrm{X} 0 \mathrm{~B} 2$ & 11/22/99 & & $0.040 \mathrm{U}$ & $-0.144 \mathrm{U}$ & $0.028 \mathrm{U}$ & $0.804 \mathrm{~J}$ & $0 \mathrm{U}$ & $0.016 \mathrm{U}$ & $2.46 \mathrm{~J}$ & $0.029 \mathrm{U}$ & 1.57 & $0.564 \mathrm{~J}$ & 0.432 & 0.344 & \\
\hline \begin{tabular}{|l|}
$99.5-$ \\
101.5 \\
\end{tabular} & \begin{tabular}{|l|}
$\mathrm{B} 0 \mathrm{X} 0 \mathrm{~B} 3$ \\
\end{tabular} & $11 / 23 / 99$ & & $0.038 \mathrm{U}$ & $-0.132 \mathrm{U}$ & $0.013 \mathrm{U}$ & $0.772 \mathrm{~J}$ & $0.005 \mathrm{U}$ & $0.117 \mathrm{~J}$ & $2.14 \mathrm{U}$ & $0.020 \mathrm{U}$ & $-0.065 \mathrm{U}$ & $0.580 \mathrm{~J}$ & $0.812 \mathrm{~J}$ & $0.534 \mathrm{~J}$ & \\
\hline $135-137$ & B0X0B4 & \begin{tabular}{|l|l|}
$11 / 30 / 99$ \\
\end{tabular} & & -- & -- & -- & 年.806 J & $-0.006 \mathrm{U}$ & $0.006 \mathrm{U}$ & & $0.019 \mathrm{U}$ & $-0.068 \mathrm{U}$ & 0.623 & - & 0.606 & \\
\hline $150-152$ & B0X0B5 & \begin{tabular}{|l|}
$12 / 01 / 99$ \\
\end{tabular} & & $-0.046 \mathrm{U}$ & $0.118 \mathrm{U}$ & $0.004 \mathrm{U}$ & (0.589 J & $-0.014 \mathrm{U}$ & $0.021 \mathrm{U}$ & $1.4 \mathrm{U}$ & $0.024 \mathrm{U}$ & $-0.027 \mathrm{U}$ & $0.484 \mathrm{~J}$ & $0.436 \mathrm{~J}$ & $0.390 \mathrm{~J}$ & \\
\hline $180-182$ & B0X0B6 & $12 / 02 / 99$ & & $-0.001 \mathrm{U}$ & $0.114 \mathrm{U}$ & $0.008 \mathrm{U}$ & $0.782 \mathrm{~J}$ & $0.004 \mathrm{U}$ & $0.012 \mathrm{U}$ & $-0.088 \mathrm{U}$ & $0.007 \mathrm{U}$ & $-0.023 \mathrm{U}$ & $0.484 \mathrm{~J}$ & $0.738 \mathrm{~J}$ & $0.387 \mathrm{~J}$ & \\
\hline 197-199 & \begin{tabular}{|l|}
$\mathrm{B} 0 \times 0 \mathrm{~B} 7$ \\
\end{tabular} & \begin{tabular}{|l|}
$12 / 07 / 99$ \\
\end{tabular} & & $0.012 \mathrm{U}$ & $-0.049 \mathrm{U}$ & $-0.016 \mathrm{U}$ & $0.377 \mathrm{~J}$ & $-0.007 \mathrm{U}$ & $0.007 \mathrm{U}$ & $1.41 \mathrm{U}$ & $0.033 \mathrm{U}$ & $-0.133 \mathrm{U}$ & $0.516 \mathrm{~J}$ & $0.243 \mathrm{~J}$ & $0.531 \mathrm{~J}$ & \\
\hline \multirow[b]{2}{*}{$\begin{array}{l}\text { Sample } \\
\text { Interval } \\
\text { (ft bgs) }\end{array}$} & \multicolumn{2}{|c|}{ SAF B99-078 } & & & & & & & & & & & & & & \\
\hline & $\begin{array}{c}\text { HEIS } \\
\text { Number }\end{array}$ & $\begin{array}{c}\text { Sample } \\
\text { Date }\end{array}$ & $\begin{array}{l}\text { CAS } \\
\text { Units }\end{array}$ & \begin{tabular}{|c|} 
Potassium \\
$4013966-$ \\
$00-2 \mathrm{pCi} / \mathrm{g}$
\end{tabular} & $\begin{array}{c}\text { Cobalt 60 } \\
10198-40- \\
0 \mathrm{pCl} / \mathrm{g}\end{array}$ & $\mid \begin{array}{c}\text { Cesium 137 } \\
10045-97-3 \\
\mathrm{pCi} / \mathrm{g}\end{array}$ & $\mid \begin{array}{l}\text { Europium } \\
15214683- \\
23-9 \mathrm{pCi} / \mathrm{g}\end{array}$ & \begin{tabular}{|c|} 
Europium \\
$15415585-$ \\
$10-1 \mathrm{pCi} / \mathrm{g}$
\end{tabular} & \begin{tabular}{|l|} 
Europium \\
$15514391-$ \\
$16-3 \mathrm{pCi} / \mathrm{g}$
\end{tabular} & \begin{tabular}{|c|} 
Radium \\
226 13982- \\
$63-3$ pCi/g \\
\end{tabular} & \begin{tabular}{|c|} 
Radium \\
$22815262-$ \\
$20-1 \mathrm{pCi} / \mathrm{g}$
\end{tabular} & \begin{tabular}{|c|} 
Uranium \\
$233 / 234$ \\
$\mathrm{U}-233 / 234$ \\
$\mathrm{pCi} / \mathrm{g}$ \\
\end{tabular} & \begin{tabular}{|c|} 
Uranium \\
235 \\
$15117-96-$ \\
$1 \mathrm{pCi} / \mathrm{g}$ \\
\end{tabular} & 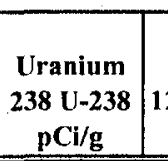 & \begin{tabular}{|c|} 
Gross \\
Alpha \\
$12587-46-1$ \\
pCi $/ \mathrm{g}$ \\
\end{tabular} & \begin{tabular}{|c|} 
Gross \\
Beta \\
$12587-47-$ \\
$2 \mathrm{pCi} / \mathrm{g}$ \\
\end{tabular} \\
\hline $35-37$ & \begin{tabular}{|c|}
$\mathrm{B} 0 \times 099$ \\
\end{tabular} & \begin{tabular}{|l|l|}
$11 / 19 / 99$ \\
\end{tabular} & & 13.7 & $0.12 \mathrm{U}$ & $0.084 \mathrm{U}$ & $0.18 \mathrm{U}$ & $0.29 \mathrm{U}$ & $0.11 \mathrm{U}$ & 0.589 & 0.536 & - & $0.19 \mathrm{U}$ & $11 \mathrm{U}$ & $3.93 \mathrm{~J}$ & $12.8 \mathrm{~J}$ \\
\hline (Dup) & \begin{tabular}{|l|}
$\mathrm{B} 0 \mathrm{X} 0 \mathrm{~B} 0$ \\
\end{tabular} & $11 / 19 / 99$ & & 12.4 & $0.071 \mathrm{U}$ & $0.062 \mathrm{U}$ & $0.16 \mathrm{U}$ & $0.24 \mathrm{U}$ & $0.16 \mathrm{U}$ & 0.396 & 0.829 & $0.407 \mathrm{~J}$ & $0.055 \mathrm{U}$ & $0.434 \mathrm{~J}$ & $7.2 \mathrm{~J}$ & $14.4 \mathrm{~J}$ \\
\hline (Split) & B0X0X4 & $111 / 19 / 99$ & & -- & $0.0155 \mathrm{U}$ & $0.0326 \mathrm{U}$ & $0.023 \mathrm{U}$ & $0.0839 \mathrm{U}$ & $0.0376 \mathrm{U}$ & - & $\overline{0.834}$ & - & -- & -- & 10.6 & 22.1 \\
\hline $50-52$ & $\mathrm{~B} 0 \mathrm{X} 0 \mathrm{~B} 1$ & $|11 / 22 / 99|$ & & 12.9 & $0.059 \mathrm{U}$ & $0.047 \mathrm{U}$ & $0.12 \mathrm{U}$ & $0.17 \mathrm{U}$ & $0.12 \mathrm{U}$ & 0.428 & 0.566 & $0.452 \mathrm{~J}$ & $0.022 \mathrm{~J}$ & $0.381 \mathrm{~J}$ & $5.61 \mathrm{~J}$ & 19.8 \\
\hline (Dup) & $\mathrm{B} 0 \times 0 \mathrm{~B} 2$ & \begin{tabular}{|l|}
$11 / 22 / 99$ \\
\end{tabular} & & 12.5 & $0.048 \mathrm{U}$ & $0.043 \mathrm{U}$ & $0.11 \mathrm{U}$ & $0.15 \mathrm{U}$ & $0.089 \mathrm{U}$ & 0.362 & 0.492 & -- & $0.15 \mathrm{U}$ & $5.4 \mathrm{U}$ & $7.62 \mathrm{~J}$ & 21.9 \\
\hline $\begin{array}{l}99.5- \\
101.5 \\
\end{array}$ & $\mathrm{~B} 0 \times 0 \mathrm{~B} 3$ & \begin{tabular}{|l|}
$11 / 23 / 99$ \\
\end{tabular} & & 13.1 & $0.030 \mathrm{U}$ & $0.029 \mathrm{U}$ & $0.070 \mathrm{U}$ & $0.10 \mathrm{U}$ & $0.067 \mathrm{U}$ & 0.564 & 0.907 & - & $0.10 \mathrm{U}$ & $3.8 \mathrm{U}$ & $9.15 \mathrm{~J}$ & 16.8 \\
\hline $135-137$ & \begin{tabular}{|l|l|} 
\\
\end{tabular} & \begin{tabular}{|l|}
$11 / 30 / 99$ \\
\end{tabular} & & 9.14 & $0.092 \mathrm{U}$ & $0.075 \mathrm{U}$ & $0.20 \mathrm{U}$ & $0.26 \mathrm{U}$ & $0.19 \mathrm{U}$ & 0.533 & 0.606 & - & $0.26 \mathrm{U}$ & $8.7 \mathrm{U}$ & 10.7 & 40.3 \\
\hline $150-152$ & B0X0B5 & \begin{tabular}{|l|l|l|} 
\\
\end{tabular} & & 10.7 & $0.014 \mathrm{U}$ & $0.12 \mathrm{U}$ & $0.29 \mathrm{U}$ & $0.34 \mathrm{U}$ & $0.28 \mathrm{U}$ & 0.311 & 0.667 & - & $0.36 \mathrm{U}$ & $15 \mathrm{U}$ & $3.02 \mathrm{~J}$ & $24.0 \mathrm{~B}$ \\
\hline $180-182$ & B0X0B6 & \begin{tabular}{|l|}
$12 / 02 / 99$ \\
\end{tabular} & & 8.51 & $0.29 \mathrm{U}$ & $0.22 \mathrm{U}$ & $0.41 \mathrm{U}$ & $0.91 \mathrm{U}$ & $0.30 \mathrm{U}$ & 0.515 & $1.2 \mathrm{U}$ & -- & $0.49 \mathrm{U}$ & $27 \mathrm{U}$ & $9.03 \mathrm{~J}$ & $8.18 \mathrm{~J}$ \\
\hline 197-199 & \begin{tabular}{|l|} 
B0X0B7 \\
\end{tabular} & \begin{tabular}{|l|}
$12 / 07 / 99$ \\
\end{tabular} & & 13.9 & $0.13 \mathrm{U}$ & $0.098 \mathrm{U}$ & $0.21 \mathrm{U}$ & $0.39 \mathrm{U}$ & $0.13 \mathrm{U}$ & 0.344 & 0.523 & -- & $0.21 \mathrm{U}$ & $14 \mathrm{U}$ & $6.58 \mathrm{~J}$ & $33.1 \mathrm{~B}$ \\
\hline \multicolumn{9}{|c|}{$\begin{array}{l}--=\text { Not analyzed. } \\
\mathrm{U}=\text { Analyzed for but not detected above the minimum detectables activity } \\
\text { in the sample. The value reported as the result. } \\
\mathrm{B}=\text { The analyte was detected in the associated QC blank and in the sample. } \\
\mathrm{J}=\text { Concentration is estimated. }\end{array}$} & $\begin{array}{l}\text { SAF }=\text { Sam } \\
\text { CAS }=\text { Che } \\
\text { Dup }=\text { Dup } \\
\text { Split }=\text { Dup }\end{array}$ & $\begin{array}{l}\text { nple Auth } \\
\text { emical At } \\
\text { olicate. } \\
\text { plicate sat }\end{array}$ & $\begin{array}{l}\text { rization Forn } \\
\text { tract Service } \\
\text { ple for qualit }\end{array}$ & $\begin{array}{l}\text { m. } \\
\text { registry nur } \\
\text { ty control pu }\end{array}$ & umber. & & & \\
\hline
\end{tabular}


Table B.8. Soil Physical Properties for Three Samples from Well 299-W26-13

\begin{tabular}{|c|c|c|c|c|c|c|c|}
\hline \multicolumn{2}{|c|}{ Sample Interval } & \multirow[b]{2}{*}{$\begin{array}{c}\text { HIES } \\
\text { umber }\end{array}$} & \multirow[b]{2}{*}{ Field pH } & \multirow[b]{2}{*}{$\begin{array}{l}\text { Moisture } \\
\text { Content } \\
(\text { wt. \%) })^{(a)}\end{array}$} & \multirow{2}{*}{$\begin{array}{c}\text { Calculated } \\
\text { Volumetric } \\
\text { Moisture } \\
\text { (vol. \%) }\end{array}$} & \multirow[b]{2}{*}{$\begin{array}{l}\text { Calculated Dry } \\
\text { Bulk Density } \\
\left(\mathrm{g} / \mathrm{cm}^{3}\right)\end{array}$} & \multirow[b]{2}{*}{$\begin{array}{c}\text { Particle Size } \\
\text { Analysis }\end{array}$} \\
\hline $\begin{array}{c}\text { Top } \\
\text { (ft bgs) }\end{array}$ & $\begin{array}{l}\text { Bottom } \\
\text { (ft bgs) }\end{array}$ & & & & & & \\
\hline 35 & 37 & $\mathrm{~B} 0 \mathrm{X} 0 \mathrm{C} 0$ & 8.61 & 6.5 & 9.64 & 1.48 & Yes \\
\hline 135 & 137 & $\mathrm{~B} 0 \mathrm{X} 0 \mathrm{Cl}$ & 8.80 & 11.6 & 24.1 & 2.07 & Yes \\
\hline 197 & 199 & $\mathrm{~B} 0 \mathrm{X} 0 \mathrm{C} 2$ & 8.85 & 2.5 & 4.9 & 1.96 & Yes \\
\hline
\end{tabular}




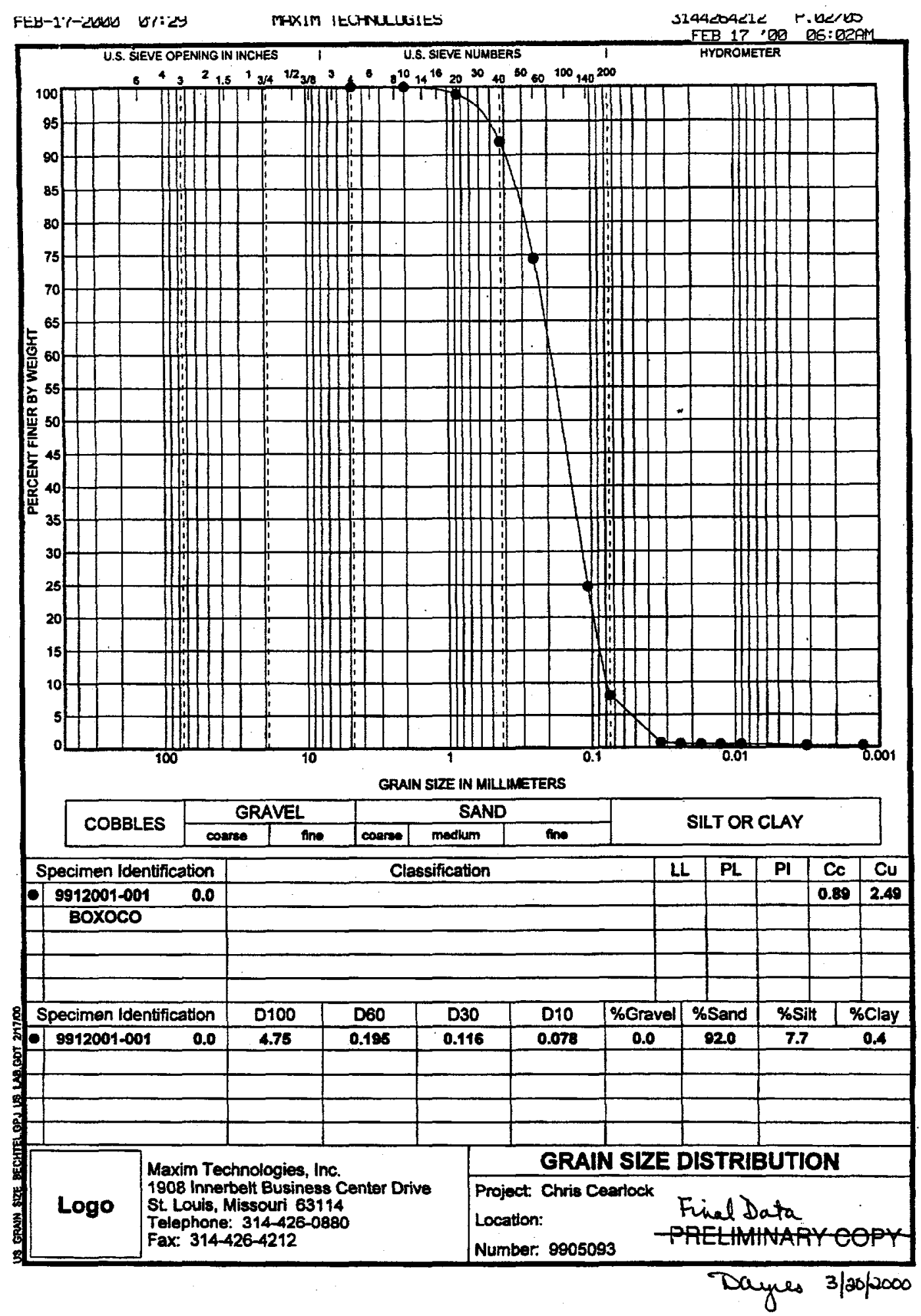

Figure B.1. Particle Size Distribution for the 35 to $37 \mathrm{ft}$ Interval in Well 299-W26-13 


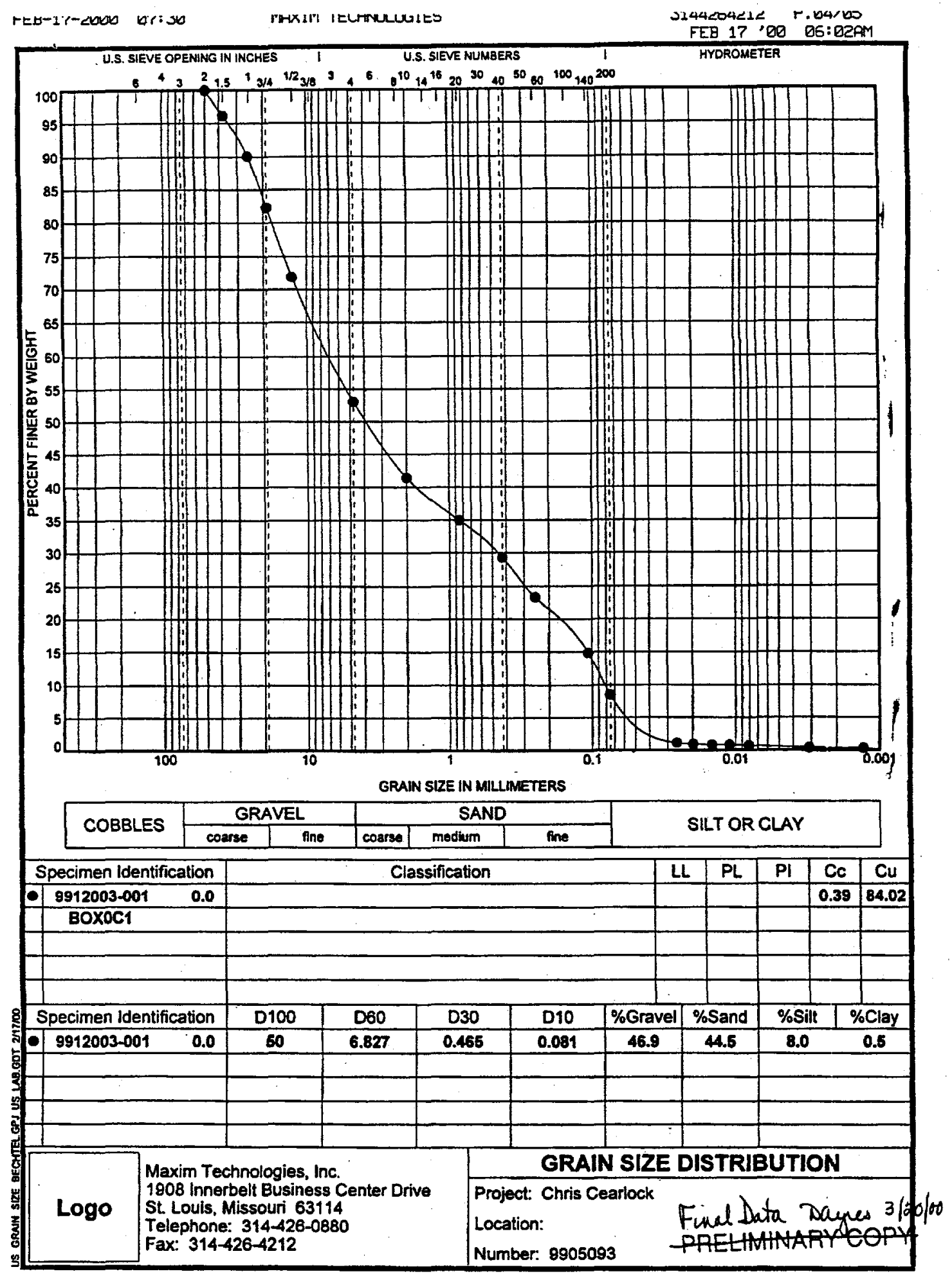

Figure B.2. Particle Size Distribution for Interval 135 to $137 \mathrm{ft}$ in Well 299-W26-13 


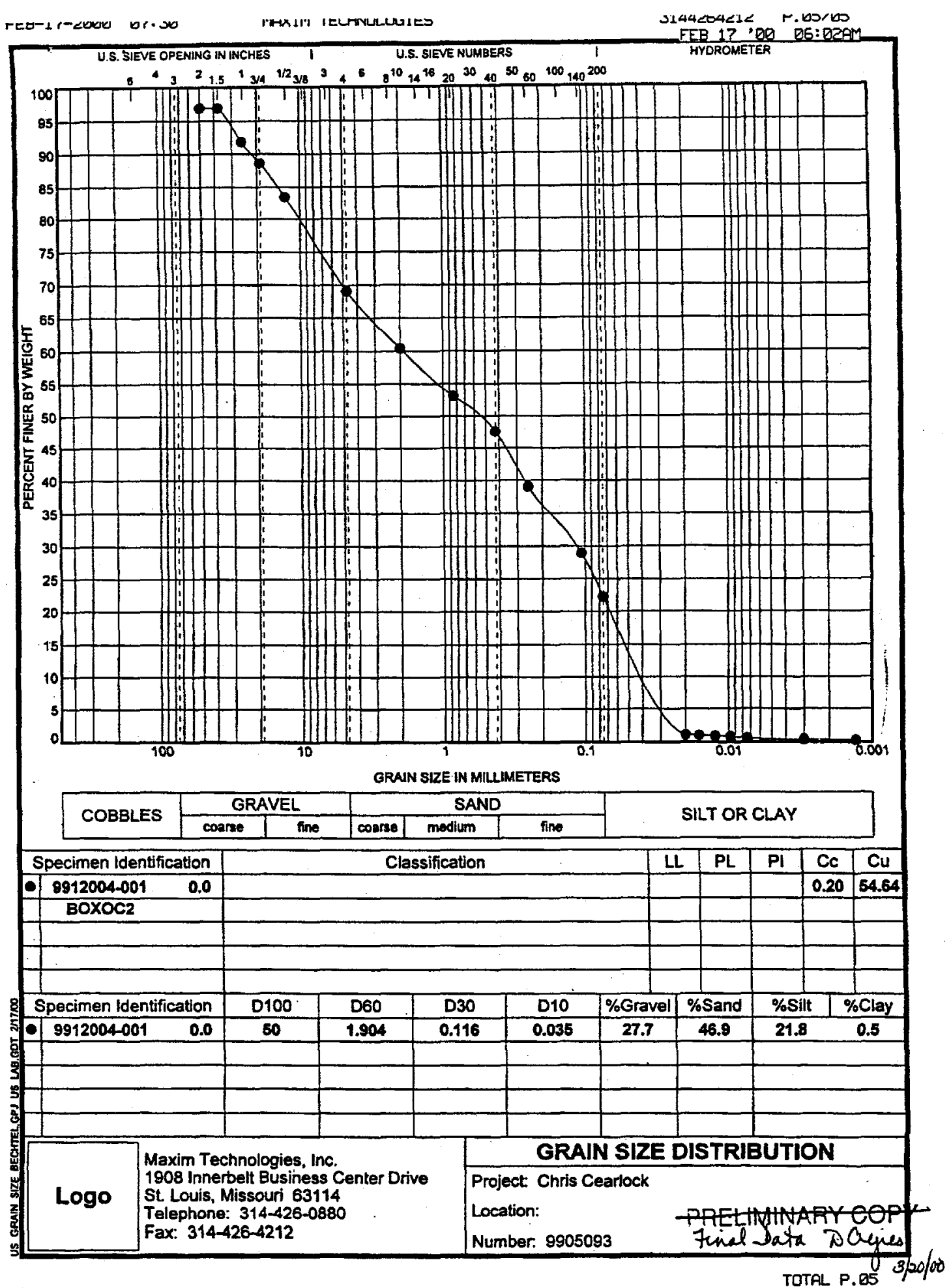

Figure B.3. Particle Size Distribution for the 197 to $199 \mathrm{ft}$ Interval in Well 299-W26-13

B. 10 


\begin{tabular}{|c|c|c|c|c|c|c|}
\hline \multicolumn{7}{|c|}{ Cम2M Hili Ranford, Inc. } \\
\hline \multirow{2}{*}{\begin{tabular}{|l} 
WELL NAME \\
TESTED BY \\
\end{tabular}} & $299-W 26-13$ & DEPTH & $199.5-200.5$ & SAMPLE\# & $B 8817-1$ & \multirow{2}{*}{\begin{tabular}{|lr} 
WELL ID\# & B8817 \\
DATE & $12 / 28 / 1999$ \\
\end{tabular}} \\
\hline & JMF $m$ & CONTACT & Dave Weekes & PHONE & $372-9582$ & \\
\hline $\begin{array}{l}\text { SAMPLE } \\
\text { WT (g) } \\
\end{array}$ & $\begin{array}{l}\text { SIEVE } \\
\text { SIZE IN. }\end{array}$ & $\begin{array}{l}\text { CUMULATIVE } \\
\text { WEIGHT(g) }\end{array}$ & $\begin{array}{l}\% \text { WEIGHT } \\
\text { RETAINED }\end{array}$ & $\begin{array}{c}\% \\
\text { PASSING }\end{array}$ & $\begin{array}{c}\text { Grain Size } \\
\text { (mm) }\end{array}$ & COMMENTS \\
\hline 703.50 & $2^{\prime \prime}$ & 0.0 & 0.0 & 100.0 & 50.00 & $\dot{-}$ \\
\hline \multirow[t]{10}{*}{ 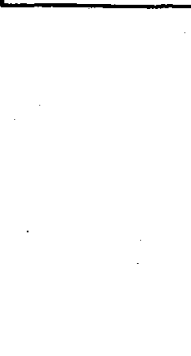 } & $1.5^{\prime \prime}$ & 94.5 & 13.4 & 86.6 & 38.10 & \\
\hline & $3 / 4^{\prime \prime}$ & 161.4 & 22.9 & 77.1 & 19.00 & \\
\hline & $3 / 8^{\prime \prime}$ & 236.9 & 33.7 & 66.3 & 9.50 & \\
\hline & $\# 4$ & 310.9 & 44.2 & 55.8 & 4.75 & \\
\hline & $\# 10$ & 371.6 & 52.8 & 47.2 & 2.00 & \\
\hline & $\# 20$ & 418.0 & 59.4 & 40.6 & 0.85 & \\
\hline & $\# 40$ & 449.0 & 63.8 & 36.2 & 0.43 & \\
\hline & $\# 60$ & 509.8 & 72.5 & 27.5 & 0.25 & \\
\hline & $\# 100$ & 556.5 & 79.1 & 20.9 & 0.150 & \\
\hline & $\# 200$ & 595.4 & 84.6 & 15.4 & 0.075 & \\
\hline
\end{tabular}

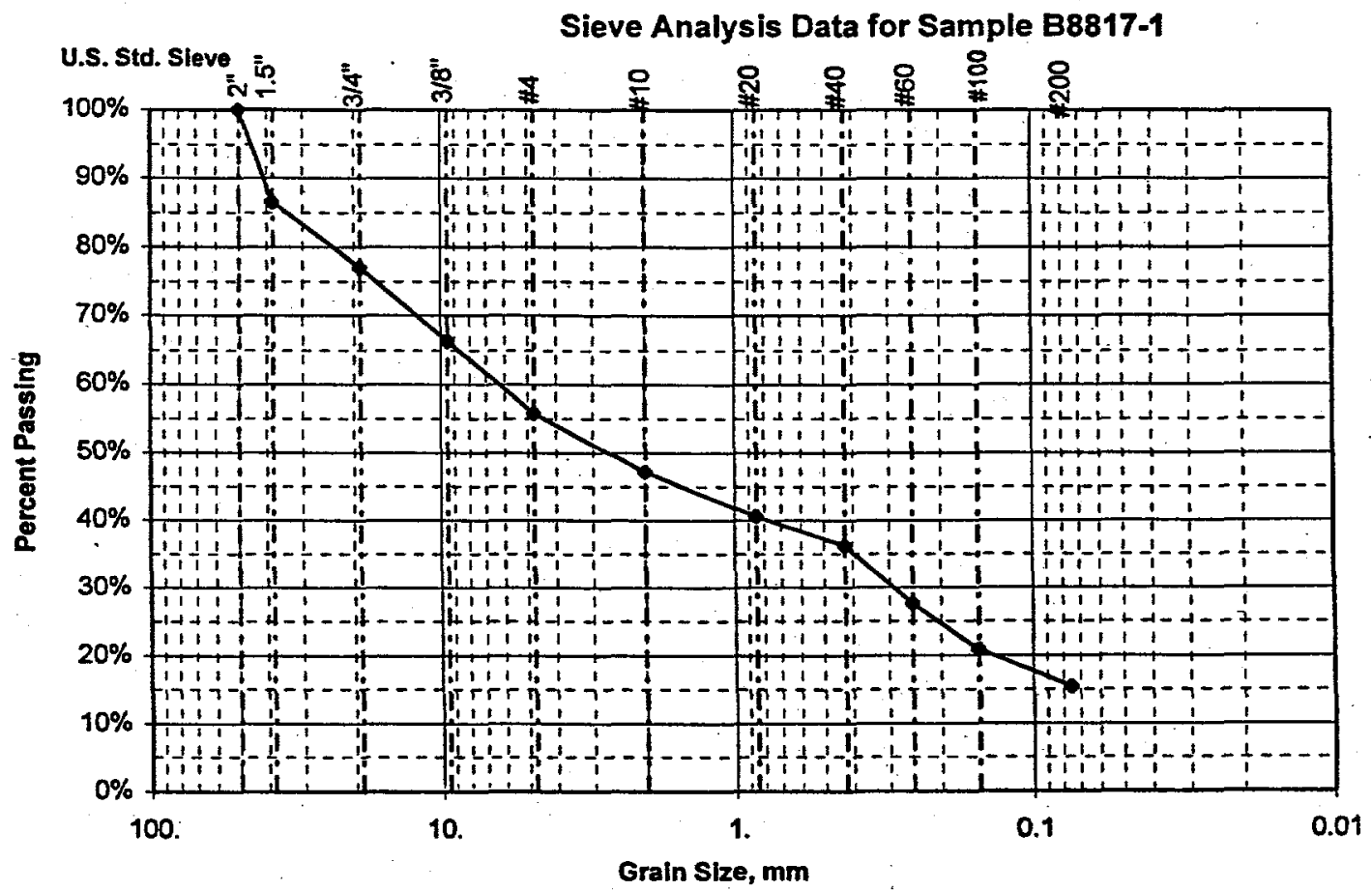

Comments: Silty Sandy Gravel

All data are accurat ${ }_{1 y}$ and completely recorded.

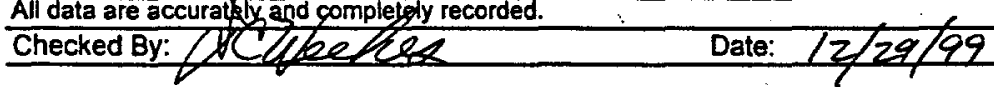

Figure B.4. Particle Size Distribution for the 199.5 to $200.5 \mathrm{ft}$ Interval in Well 299-W26-13 


\begin{tabular}{|c|c|c|c|c|c|c|}
\hline & & $\begin{array}{l}\text { PLMPIITHant } \\
\text { SIEVE ANAl }\end{array}$ & $\begin{array}{l}\text { ord, Ine. } \\
\text { YSIS }\end{array}$ & & \\
\hline WELL NAME & 299-W26-13 & DिEPTH & $276^{\prime}-277^{\prime}$ & SAMPLE\# & B8817-2 & WELLLID\# \\
\hline TESTED BY & JMF 7777 & CONTACT & Dave Weekes & PHONE & $372-9582$ & DATE $12 / 28 / 1999$ \\
\hline & 72 & & & & & \\
\hline \begin{tabular}{|l} 
SAMPLE \\
$W T(g)$ \\
\end{tabular} & $\begin{array}{l}\text { SIEVE } \\
\text { SIZE IN. }\end{array}$ & $\begin{array}{l}\text { CUMULATIVE } \\
\text { WEIGHT(g) }\end{array}$ & $\begin{array}{l}\text { \% WEIGHT } \\
\text { RETAINED }\end{array}$ & $\begin{array}{c}\% \\
\text { PASSING }\end{array}$ & $\begin{array}{c}\text { Grain Size } \\
\text { (mm) }\end{array}$ & COMMENTS \\
\hline 956.70 & $2^{\prime \prime}$ & 0.0 & 0.0 & 100.0 & 50.00 & \\
\hline & $1.5^{n}$ & 182.2 & 19.0 & 81.0 & 38.10 & \\
\hline & $3 / 4^{\prime \prime}$ & 440.0 & 46.0 & 54.0 & 19.00 & \\
\hline & $3 / 8^{11}$ & 587.1 & 61.4 & 38.6 & 9.50 & \\
\hline . & \#4 & 653.2 & 68.3 & 31.7 & 4.75 & \\
\hline & $\# 10$ & 710.1 & 74.2 & 25.8 & 2.00 & \\
\hline & $\# 20$ & 749.2 & 78.3 & 21.7 & 0.85 & . \\
\hline & $\# 40$ & 778.2 & 81.3 & 18.7 & 0.43 & \\
\hline & $\# 60$ & 822.6 & 86.0 & 14.0 & 0.25 & \\
\hline & $\# 100$ & 858.2 & 89.7 & 10.3 & 0.150 & \\
\hline & $\# 200$ & 887.9 & 92.8 & 7.2 & 0.075 & \\
\hline
\end{tabular}

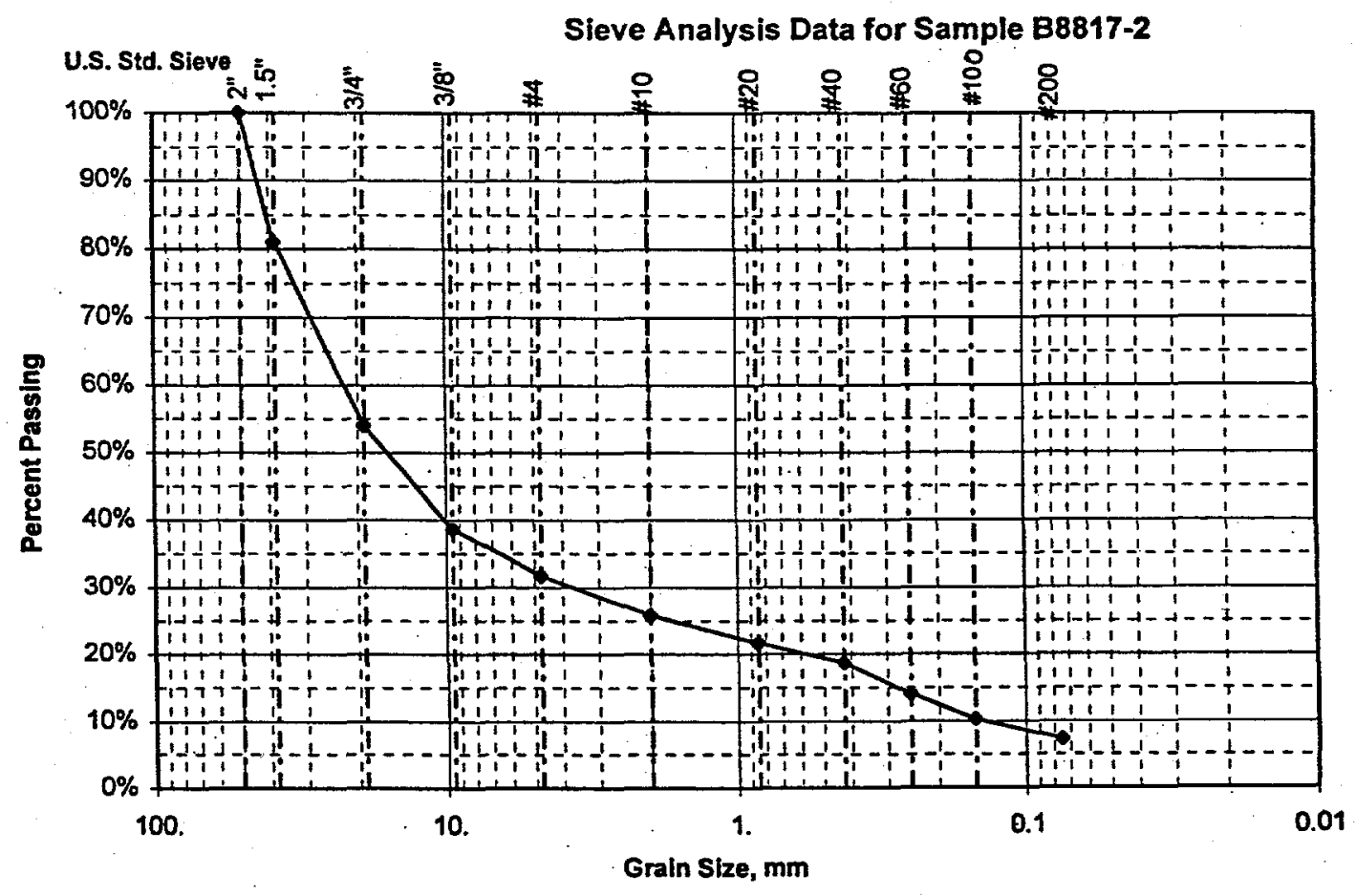

Comments: Sandy Gravel

All data are accurately and completely recorded.

Checked By: DC qbe her Date: 12/29/99

Figure B.5. Particle Size Distribution for the 216 to $217 \mathrm{ft}$ Interval in Well 299-W26-13

B. 12 
CH2M Hill Hanford, Inc.

SIEVE ANALYSIS

\begin{tabular}{|c|c|c|c|c|c|c|}
\hline \multirow{2}{*}{$\begin{array}{l}\text { WELL NAME } \\
\text { TESTED BY }\end{array}$} & $299-w / 46-13$ & DEPTH & $231^{1}-233^{\top}$ & SAMPLE\# & B8817-3 & \multirow{2}{*}{ 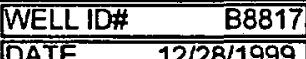 } \\
\hline & JMF WII & CONTACT & Dave Weekes & PHONE & $372-9582$ & \\
\hline & & & & & & \\
\hline $\begin{array}{l}\text { SAMPLE } \\
\text { WT (g) }\end{array}$ & $\begin{array}{l}\text { SIEVE } \\
\text { SIZE IN. }\end{array}$ & $\begin{array}{l}\text { CUMULATIVE } \\
\text { WEIGHT(g) }\end{array}$ & $\begin{array}{l}\% \text { WEIGHT } \\
\text { RETAINED }\end{array}$ & $\begin{array}{c}\% \\
\text { PASSING }\end{array}$ & $\begin{array}{c}\text { Grain Size } \\
(\mathrm{mm})\end{array}$ & COMMENTS \\
\hline 1209.60 & $2^{\prime \prime}$ & 306.1 & 25.3 & 74.7 & 50.00 & $\cdot$ \\
\hline & $1.5^{\prime \prime}$ & 420.7 & 34.8 & 65.2 & 38.10 & • \\
\hline & $3 / 4^{\prime \prime}$ & 701.0 & 58.0 & 42.0 & 19.00 & \\
\hline & $3 / 8^{\prime \prime}$ & 949.6 & 78.5 & 21.5 & 9.50 & \\
\hline & \#4 & 1008.2 & 83.3 & 16.7 & 4.75 & \\
\hline & $\# 10$ & 1021.3 & 84.4 & 15.6 & 2.00 & \\
\hline & \#20 & 1026.9 & 84.9 & 15.1 & 0.85 & \\
\hline & $\# 40$ & 1042.6 & 86.2 & 13.8 & $\overline{0.43}$ & \\
\hline & $\# 60$ & 1121.9 & 92.7 & $\overline{7.3}$ & 0.25 & \\
\hline & $\# 100$ & 1174.2 & 97.1 & 2.9 & 0.150 & \\
\hline & $\# 200$ & 1194.8 & 98.8 & 1.2 & 0.075 & \\
\hline
\end{tabular}

\section{Sieve Analysis Data for Sample B8817-3}

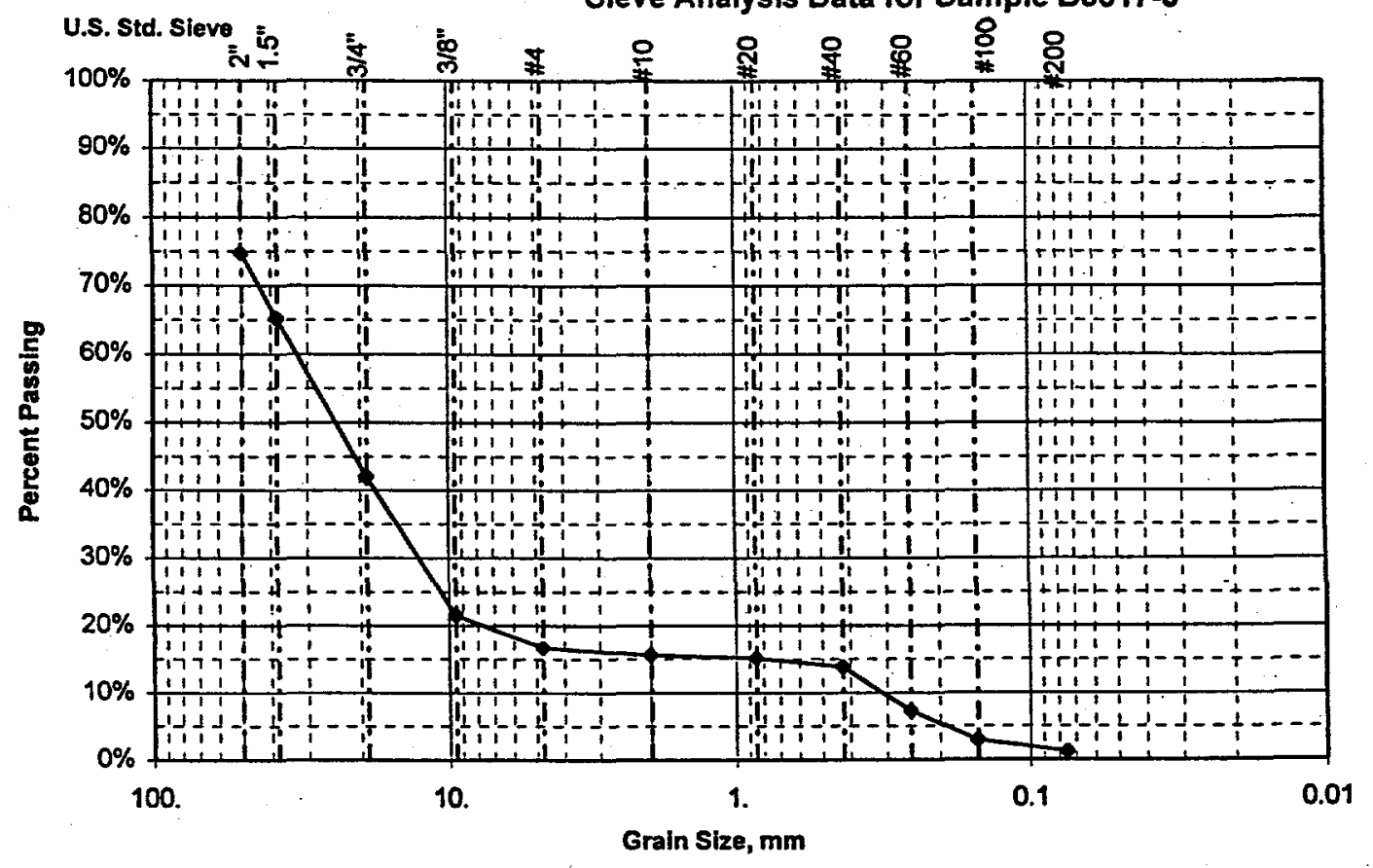

Comments: Gravel

All cata are accurajely and completely recorded.

Checked By: ACQteeles Date: $12 / 29799$

Figure B.6. Particle Size Distribution for the 231 to $233 \mathrm{ft}$ Interval in Well 299-W26-13 


\section{Appendix C}

\section{Borehole Geophysical Logs}




\section{Appendix C}

\section{Borehole Geophysical Logs}

This appendix contains the borehole geophysical logs obtained from borehole 299-W26-13. The gamma-ray log was run and analyzed by MACTEC-ERS, Inc. The neutron-neutron moisture log was run and analyzed by Waste Management Federal Services, Inc. Included with the logs are Log Header sheets and Log Analysis Summary Reports. 


\section{Spectral Gamma-Ray Borehole Log Data Report}

Borehole 299-W26-13

\section{Borehole Information}

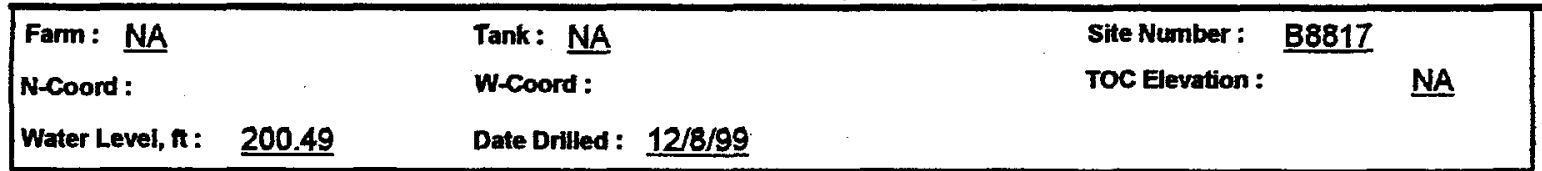

\section{Casing Record}

\begin{tabular}{|c|c|c|}
\hline Steel thread & Thickness, in.: 0.500 & ID, in. : 11 \\
\hline Top Depth, $\mathrm{n}:$ : $\underline{\underline{0}}$ & Bottom Depth, r.: $\quad \underline{36}$ & \\
\hline Steel thread & Thickness, in.: 0.500 & ID, in. : \\
\hline
\end{tabular}

Top Depth, $\pi$ : $\quad \underline{0} \quad$ Bottom Depth, $\mathrm{t}$ : $\underline{\mathbf{2 4 0}}$

Cement Bottom, nt. $\quad \underline{0} \quad$ Cement Top, ft. : $\quad \underline{0}$

Borehole Notes:

This borehole was drilled during December 1999 to a depth of $240.7 \mathrm{ft}$. A nominal 11-in.-diameter casing was used from the ground surface to $36.3 \mathrm{ft}$, with an 8-in.-diameter casing set from the ground surface to $240.0 \mathrm{ft}$. The casing strings used in the borehole were threaded and flush-jointed. The borehole was uncased from 240.0 to $240.7 \mathrm{ft}$. Measured wall thickness for both the 11- and 8 -in.-diameter casings was $0.5 \mathrm{in}$. Grout was not present between the casings, which were set flush with the ground surface. The total depth achieved with the logging system was $\mathbf{2 4 0 . 5} \mathrm{ft}$. The ground surface was used as the zero reference $(0 \mathrm{ft})$ for all logging depths. The ground water level was measured at $200.49 \mathrm{ft}$.

\section{Equipment Information}

\begin{tabular}{|c|c|c|}
\hline $\begin{array}{l}\text { Logging System : } 2 \mathrm{~B} \\
\text { Calibration Date : } 10 / 1999\end{array}$ & $\begin{array}{l}\text { Detector Type: } \quad \text { HPGe } \\
\text { Ceffbration Reference : GJOHAN-26 }\end{array}$ & $\begin{array}{l}\text { Detector Efficiency: } \quad \frac{35.0 \%}{\text { Logging Procecture : MAC-VZCP 1.7.10-1 }} \\
\text { MA }\end{array}$ \\
\hline
\end{tabular}

\section{Logging Information}

\begin{tabular}{|c|c|c|}
\hline $\begin{array}{lr}\text { Log Run Number : } & 1 \\
\text { Start Depth, } \pi .: & 240.5 \\
\text { Finish Depth, nt: } & 119.0\end{array}$ & $\begin{array}{l}\text { Log Run Date : } \quad \frac{12 / 09 / 1999}{\text { Counting Thme, sec.: }} \quad \underline{100} \\
\text { MSA Interval, th: } \quad \quad \underline{0.5}\end{array}$ & $\begin{array}{l}\text { Logging Engineer:. } \\
\text { Bob Spatz } \\
\text { Log Speed, ttmin.: } \quad \text { n/a }\end{array}$ \\
\hline 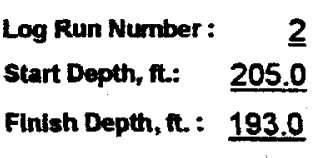 & $\begin{array}{l}\text { Log Run Date : } \quad \frac{12 / 09 / 1999}{\text { Counthg Thme, sec.: }} \quad \underline{100} \\
\text { MSA Interval, th. : }\end{array}$ & $\begin{array}{l}\text { Logging Engineer: } \quad \text { Bob Spatz } \\
\text { LR: } \underline{L} \quad \text { Shleld : } \underline{N} \\
\text { Log Speed, nimin.: } \quad \underline{\text { n/a }}\end{array}$ \\
\hline
\end{tabular}




\section{Spectral Gamma-Ray Borehole Log Data Report}

Borehole 299-W26-13

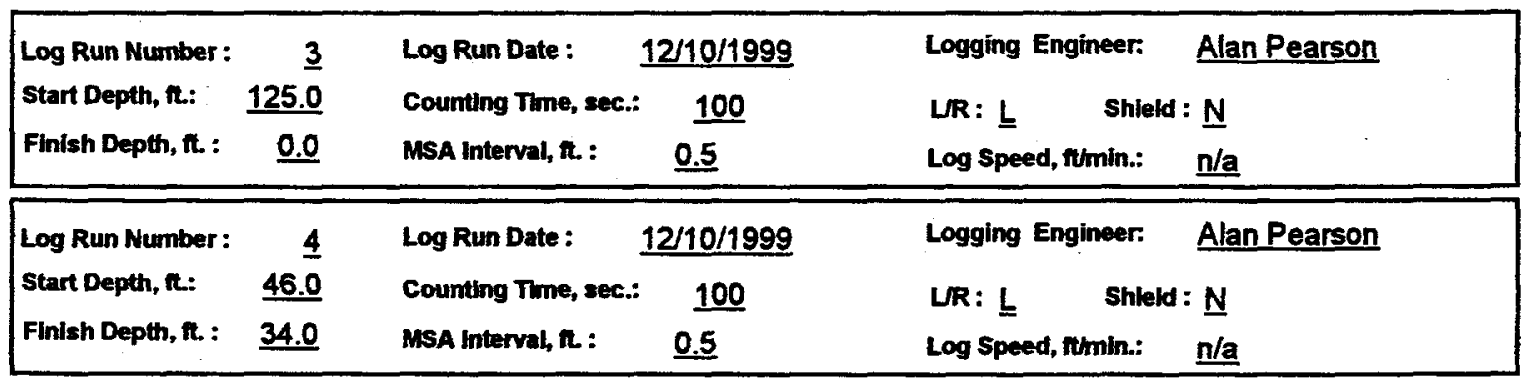

\section{Logaing Operation Notes:}

This borehole was logged in four log runs. Log runs 1 and 2 were completed on December 9, 1999 inside the 8-in.-diameter casing. Log run 1 covered the interval from 240.5 to $119.0 \mathrm{ft}$. Log run 2 was a repeat section from 205.0 to $193.0 \mathrm{ft}$. Log runs 3 and 4 were completed on December 10, 1999 inside the 8- and 11-in.diameter casings. Log run 3 covered the interval from the ground surface to $125.0 \mathrm{ft}$. $\log$ run 4 was a repeat section from 34.0 to $46.0 \mathrm{t}$. In addition, there is a depth overlap of log data (6 ft) where $\log$ runs 1 and 3 were both conducted ( 119.0 to $125.0 \mathrm{ft}$ ). The total logging footage, including the repeat sections, measured 270.5 ft.

\section{Analysis Information}

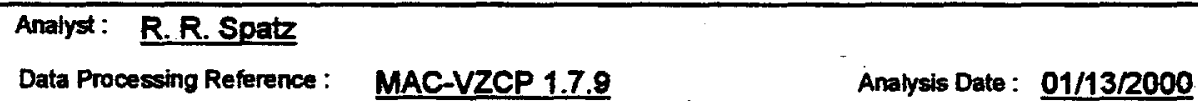

\section{Analysis Notes:}

The pre-survey and post-survey field verification for each logging run met the acceptance criteria established for peak shape and system efficiency. The energy calibration and peak-shape calibration from the accepted calibration spectrum that most closely matched the field data were used to establish the peak resolution and channel-to-energy parameters used in processing the spectra acquired during the logging operation.

The thicknesses of the different-sized casings were measured on site with a micrometer and are presented in the casing record. Casing corrections for each applicable thickness were determined and applied to the data at the appropriate depth intervals. In addition, a correction for water encountered in the borehole was applied to the data collected below $200.49 \mathrm{ft}$ in depth. These corrections are applied to the raw data recorded in counts per second to derive final radionuclide concentrations.

Shape factor analysis was not applied to the SGLS data because man-made radionuclides were not detected in this borehole.

\section{Log Plot Notes:}

Separate log plots are presented to show the man-made (none detected) and naturally occurring radionuclides (K-40, U-238, and Th-232). These plots show the naturally occurring radionuclides which can be used for lithology interpretation and bed boundary identification. The headings of the plots identify the specific gamma rays used to calculate the concentrations. The naturally occurring radionuclide plots are composites of log runs 


\section{Spectral Gamma-Ray Borehole Log Data Report}

\section{Borehole 299-W26-13}

gamma derived from the spectral data. This plot is a composite of log data collected from log runs 1 and 3 .

On the repeat $\log$ plots, repeat $\log$ data from runs 2 and 4 are compared with runs 1 and 3 , respectively.

For U-238 concentrations calculated between the ground surface and $36.5 \mathrm{ft}$, the 1764.51-keV bismuth-214 (Bi214) gamma ray was substituted for the 609.32-keV Bi-214 gamma ray. For many of those spectra in that interval the 609.32-keV Bi-214 gamma ray was not detected because of heavy gamma-ray attenuation associated with the 1.0-in. double casing wall thickness.

Where shown, the error bars on the plots show the statistical uncertainties for the measurements as 95-percent (2-sigma) confidence intervals. Error bars shown on the log plot include the error associated with calibration measurements, and errors associated with the casing and water corrections, as well as counting error. Open circles on the plots give the minimum detection level (MDL). The MDL of a radionuclide represents the lowest concentration at which positive identification of a gamma-ray peak is statistically defensible.

\section{Results/nterpretations:}

Man-made radionuclides were not detected in this borehole.

The naturally occurring radionuclides show several changes in the KUT concentrations that indicate lithology changes and bed boundaries.

Error bars representing the 2-sigma uncertainty in the measurements increase above the 36- $t$ depth and below the 200-ft depth as a result of additional error introduced by the correction for double casing and the presence water; respectively.

The repeat log plots show good repeatability for the calculated concentrations and depth. 


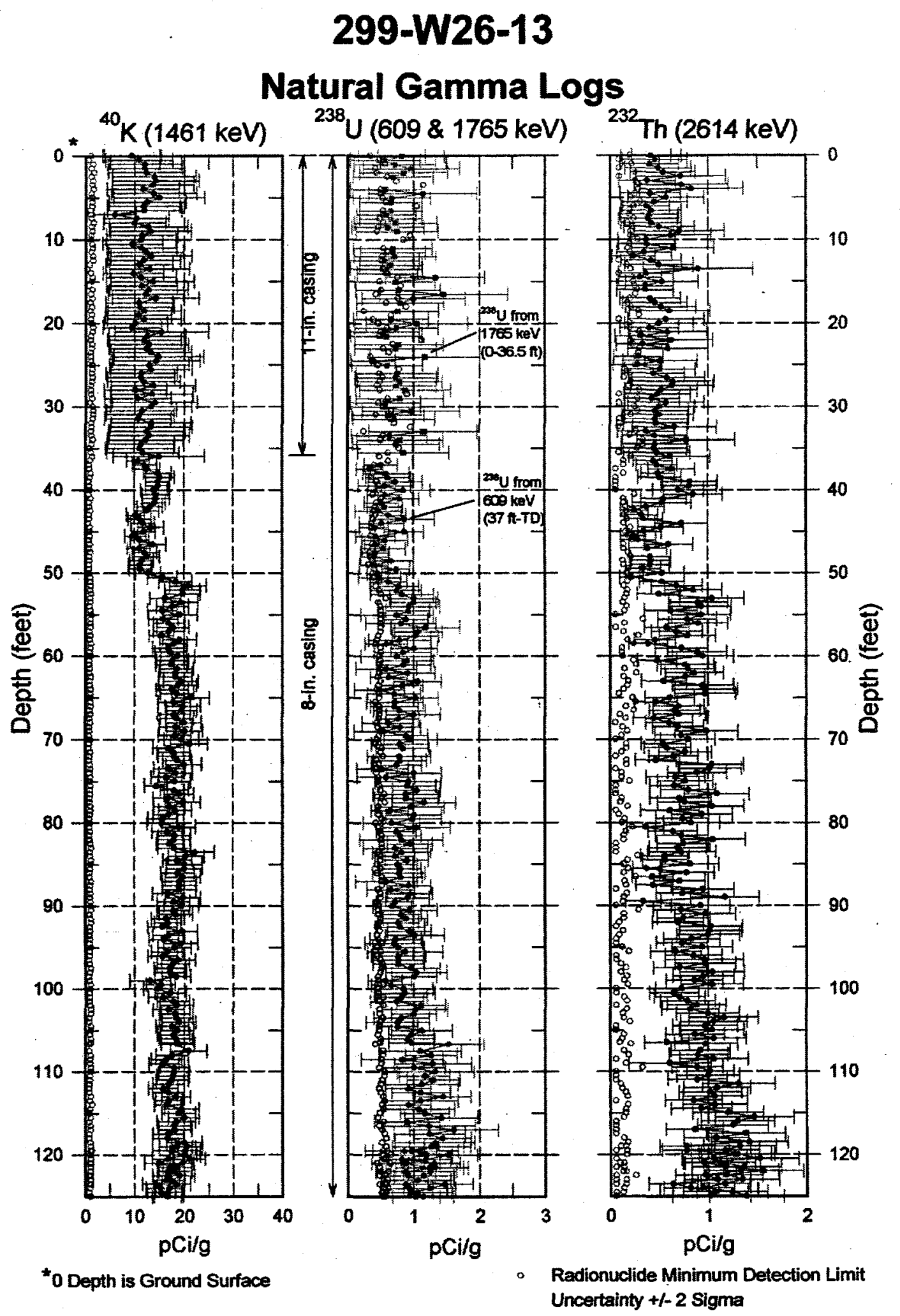

C. 5 


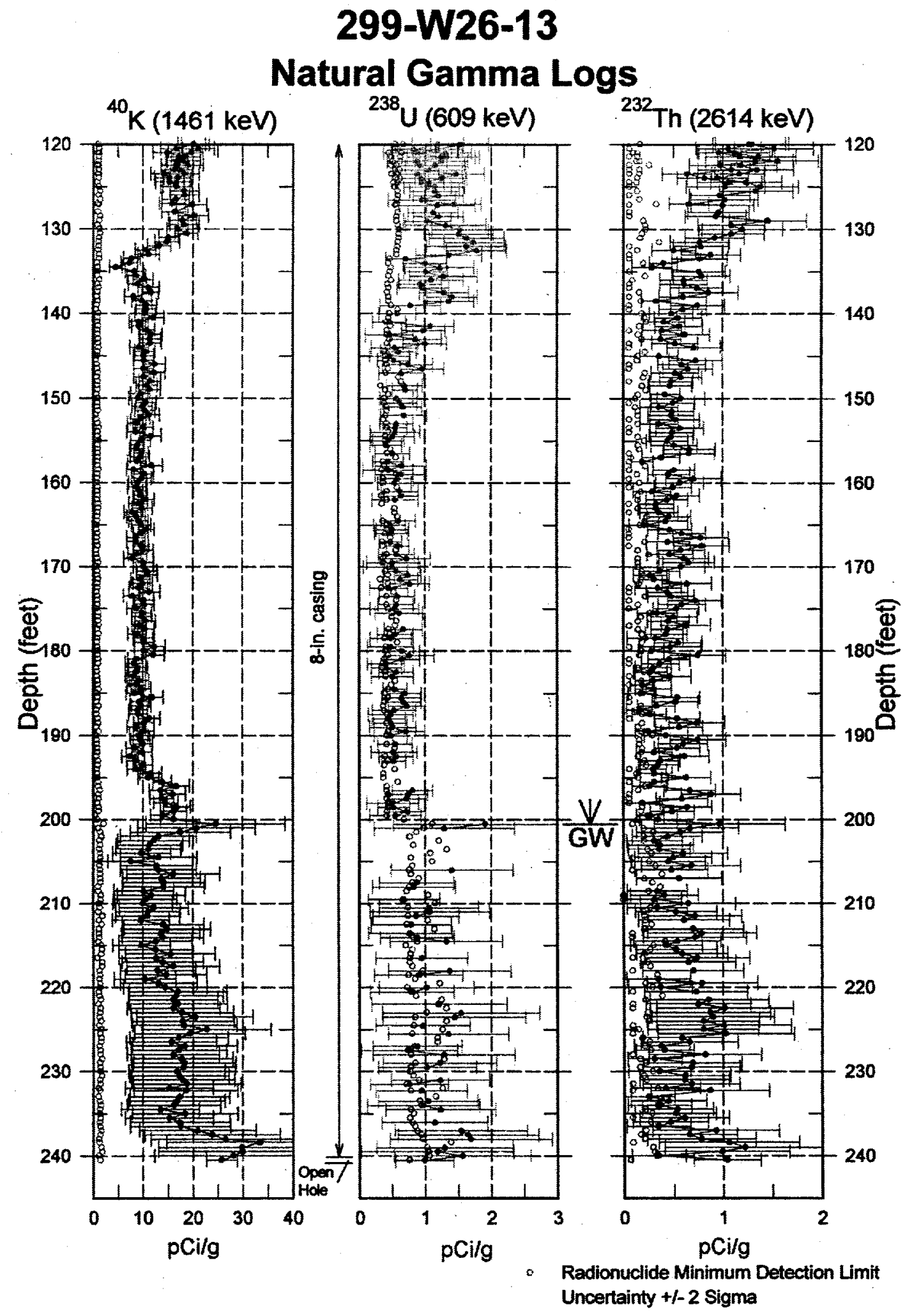

C. 6 


\section{9-W26-13 Combination Plot}

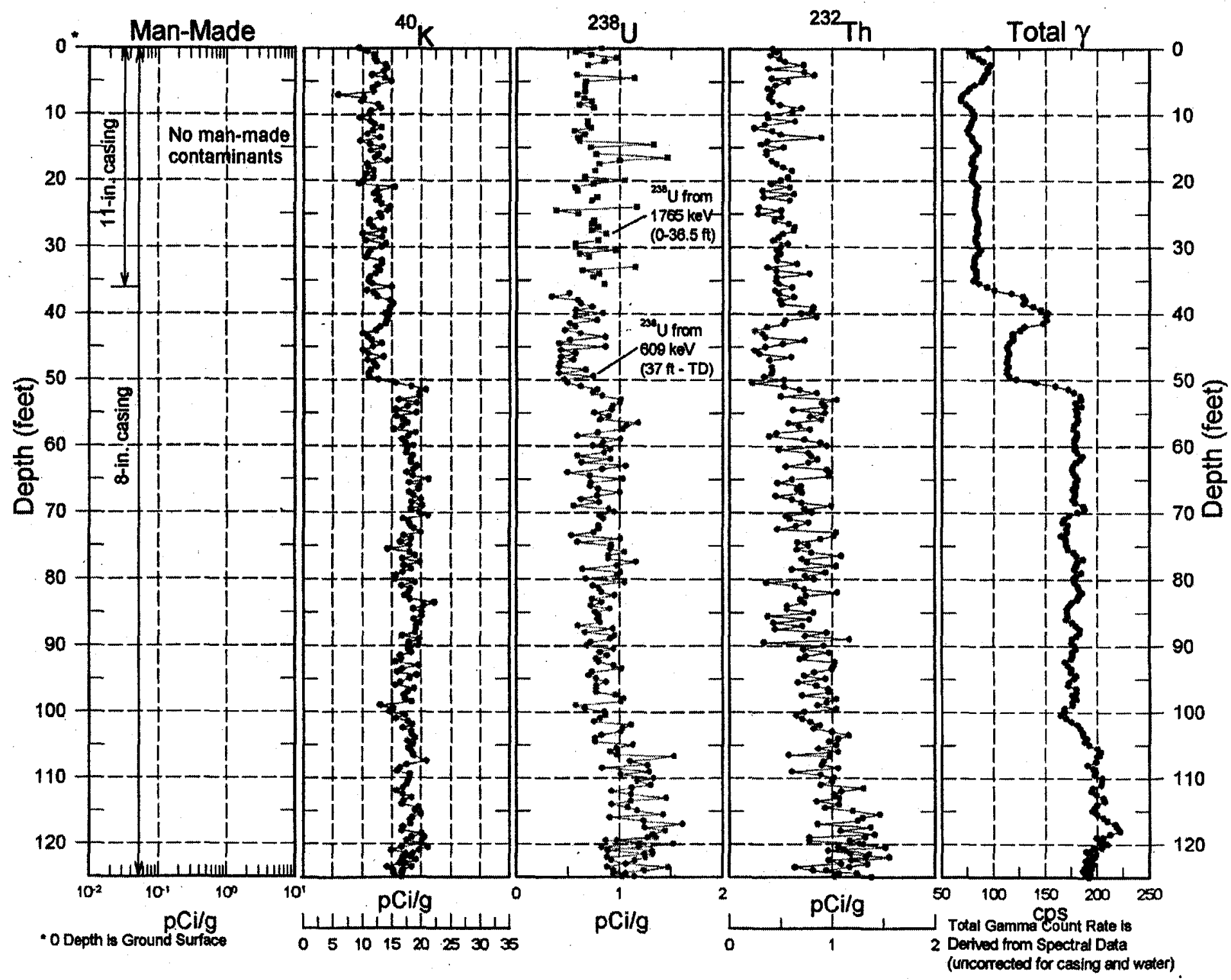




\section{9-W26-13 Combination Plot}

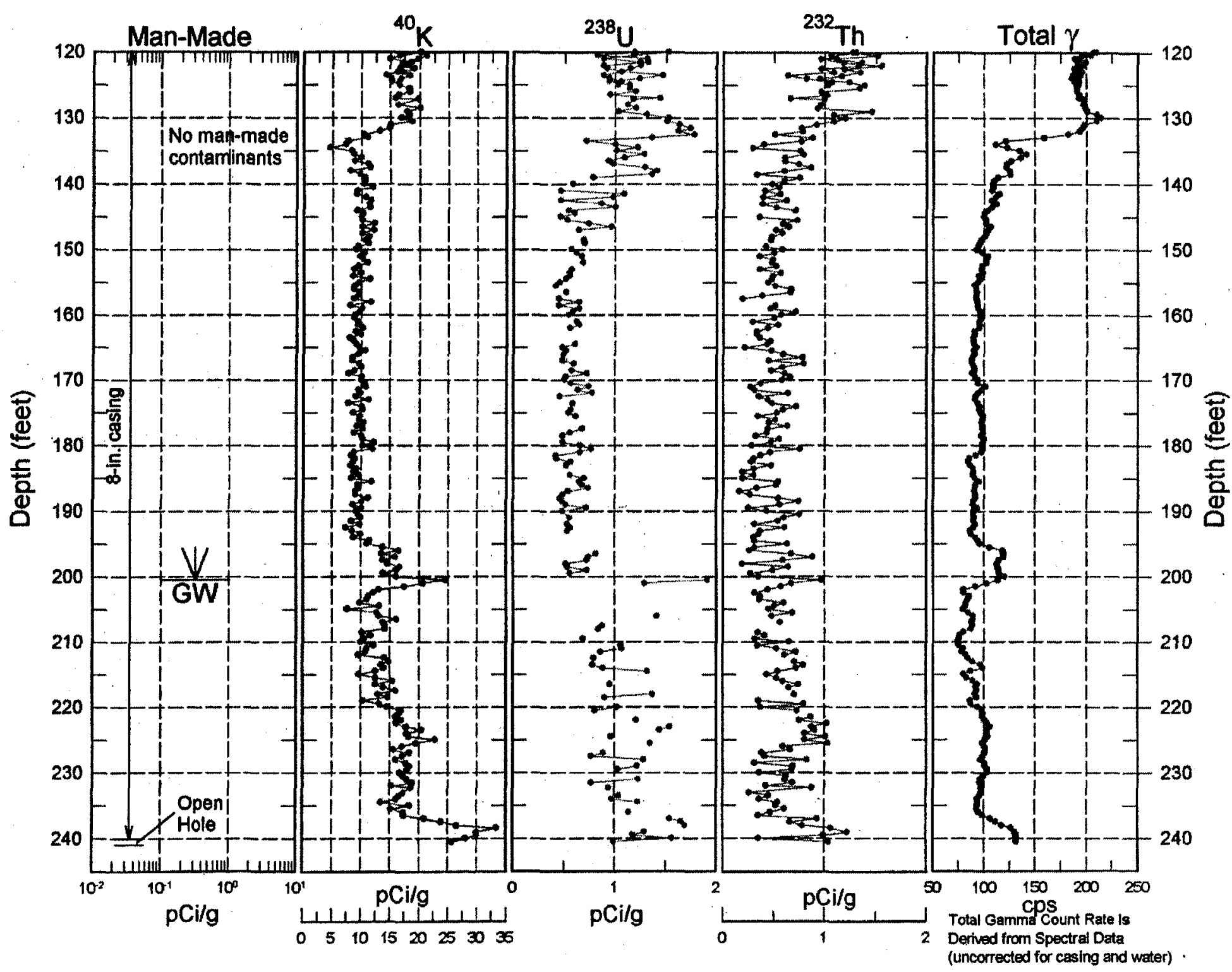


299-W26-13

\section{Repeat Section of Natural Gamma Logs}

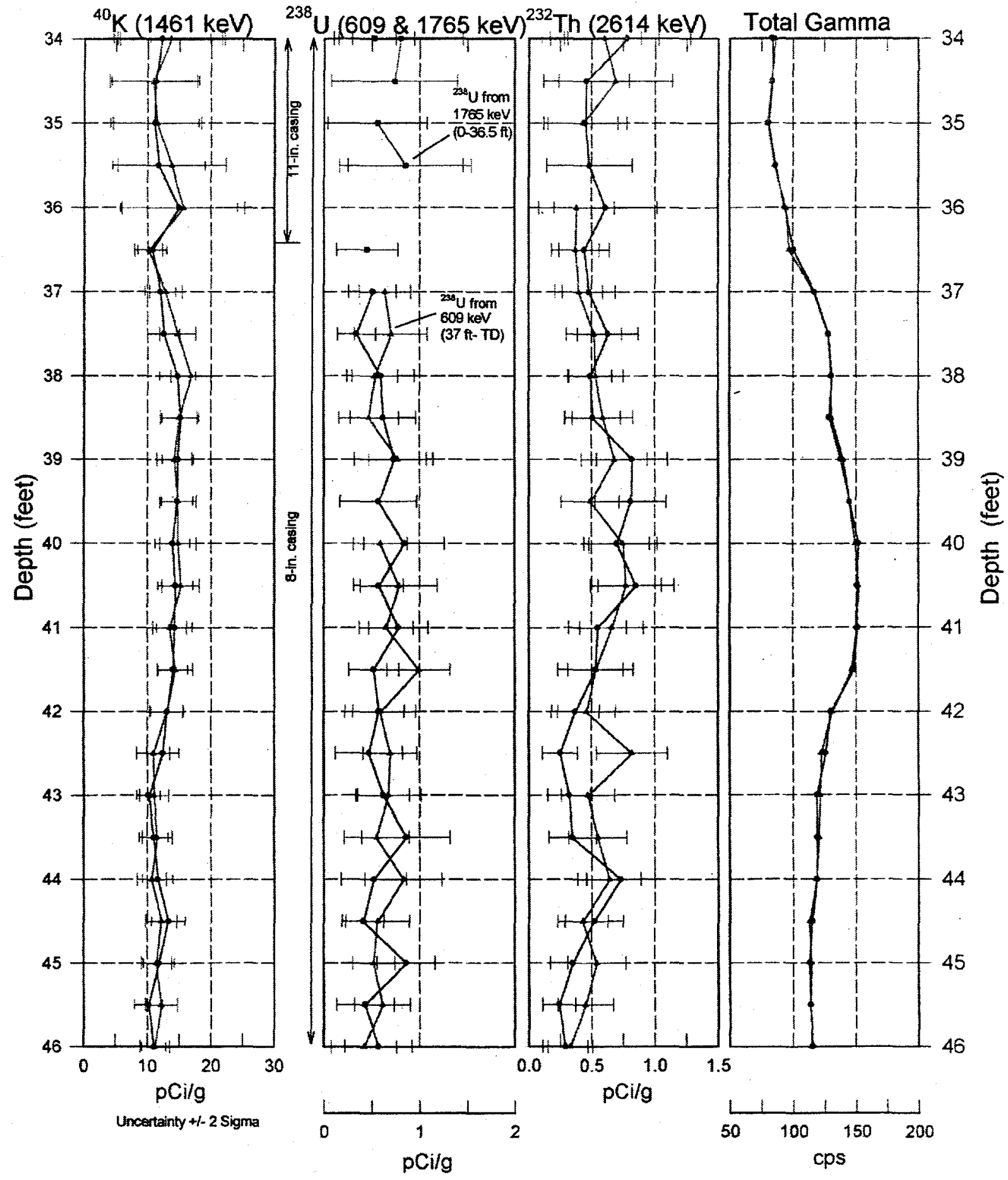




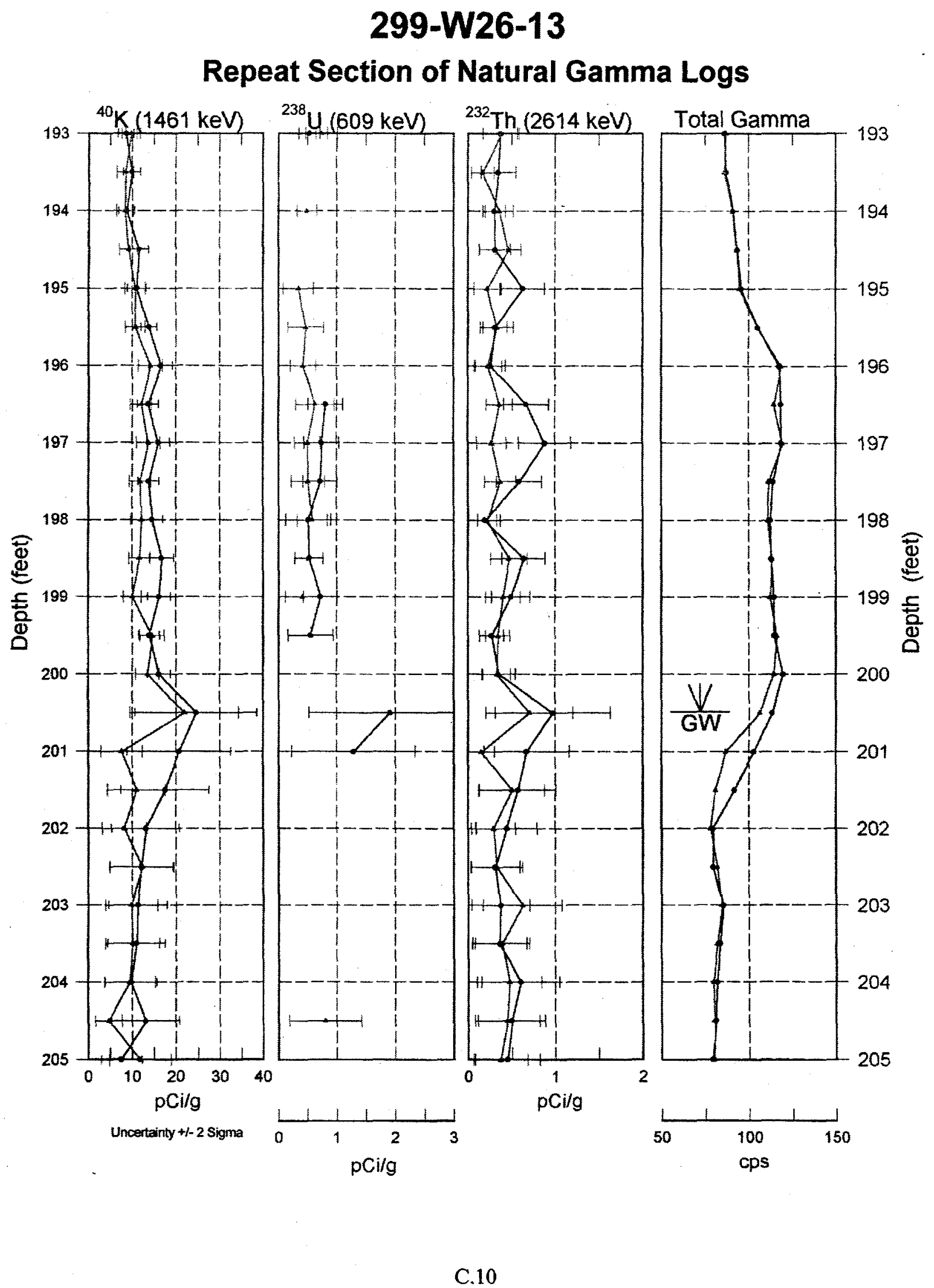




\section{Waste Management Technical Services MACTEC}

\section{Report of Geophysical Logging for the 200-CS-1/RCRA Project}

This report presents a summary of spectral gamma and neutron-neutron moisture logging that was conducted in borehole B8817 at the 216-S-10 Pond southwest of the 200 West Area. This borehole was utilized for characterization of this waste facility, and at the completion of drilling a monitoring well with the designation $299-$ W26-13 was constructed.

\section{Summary of Logging Operations}

\section{Spectral Gamma Surveys}

A spectral gamma survey was conducted in borehole B8817 utilizing a MACTEC operated logging unit designated Gamma 2B. The borehole was logged in four individual log runs in 11 -in.- and 8-in.-diameter casings when drilling was complete. Two of these log runs were repeat surveys that were logged to assess the repeatability of the measurements. The depth intervals logged in each log run are indicated on the Spectral Gamma-Ray Borehole Log Data Report for borehole B8817.

All spectral gamma measurements were acquired in a move-stop-acquire mode, at a measurement acquisition rate of 100 seconds (s) live time per $0.5 \mathrm{ft}$ of depth. In the move-stop-acquire mode the logging tool is stationary at each $0.5 \mathrm{ft}$ depth increment throughout the depth interval logged. In the live time acquisition mode, system dead time is accounted for in the acquisition time for each spectrum, e.g., a $100 \mathrm{~s}$ live time acquisition with 50 percent dead time would result in a real time measurement of $150 \mathrm{~s}$.

The zero depth reference for all the spectral gamma logging surveys is ground surface. The zero position of the logging is the center of the germanium detector, which is about $5 \mathrm{in}$. above the bottom of the logging tool. This location is scribed into the tool housing and ensures consistent tool positioning.

The pre- and post- survey field verification spectra met the acceptance criteria established for the peak shape and detector efficiency, confirming that the spectral gamma logging system was operating within specifications. The energy calibration and peak-shape calibration from these spectra were used to establish the channel-to-energy parameters that were used to process the spectra acquired during the log surveys.

\section{Neutron-Neutron Survey}

Neutron-neutron moisture logging was conducted in borehole B8817 utilizing a logging system operated by Waste Management Technical Services that is designated Radionuclide Logging System unit 1 (RLS1). The neutron-neutron moisture-logging tool is calibrated in moisture models with 6-in.- and 8-in.diameter casing configurations. Since there is no direct calibration standard for 11-in.-diameter casing, the section of well B8817 with 11-in.-diameter casing (ground surface to a depth of $36.3 \mathrm{ft}$ ) was not logged. The section of the borehole with 8-in.-diameter casing was logged with the moisture tool from the bottom of the $11 \mathrm{in}$.-diameter casing to groundwater ( 36.3 to $200.5 \mathrm{ft}$ ). The interval logged and the repeat survey interval are indicated on the Borehole Survey Data Sheet for borehole B8817.

The neutron-neutron moisture data were collected at $0.25-\mathrm{ft}$ depth increments. The data were acquired in a continuous logging mode at a logging speed of 1.0 feet per minute $(\mathrm{ft} / \mathrm{min})$; this logging speed resulted 
in a data sample time of 15 seconds real (clock) time. Given the count rate of the instrument and sample time, the statistical accuracy is within the specified precision. The guidelines for conducting the neutronneutron survey are outline in WMNW-CM-004, Operational Environmental Monitoring, Section 22.0, Rev. 0, Vadose Neutron Moisture Logging Procedure.

The neutron-neutron moisture logging tool is depth zeroed at the top of the cablehead (the cable to logging tool connection) to minimize exposure to the logging operator or other personnel to the americium-beryllium $(\mathrm{AmBe})$ neutron source. The distance from the top of the cablehead to the scribe line zero of the neutron instrument (half distance between the source and the detector) is entered for the depth datum of the borehole. The neutron-neutron log survey zero reference is ground surface.

The source shield is used as a pre- and post- survey field verification of proper instrument performance. Consistent results for the pre- and post-survey verifications that were conducted before and after the moisture survey was conducted in borehole B8817 indicated the system was operating properly.

\section{Data Processing}

The spectra acquired in borehole B8817 during the spectral gamma survey were processed utilizing a variety of software to calculate concentrations of individual gamma-emitting radionuclides. Details regarding the algorithms used to calculate the concentrations and their application are presented in the Hanford Tank Farms Vadose Zone, Data Analysis Manual (MAC-VZCP-1.7.9, Rev. 1).

Equipment calibrations are conducted annually and the data acquired during the calibrations are used to derive the factors that convert measured peak area countrate into radionuclide concentrations in picocuries per gram $(\mathrm{pCi} / \mathrm{g})$. The calibration dates and calibration reports that present the mathematical functions that determine the calibration factors for the logging unit utilized for logging for this project are referenced on the log headers for each borehole/well log. The details of the base calibration are discussed in Vadose Zone Characterization Project at the Hanford Tank Farms, Calibration of Two Spectral Gamma-Ray Logging Systems for Baseline Characterization Measurements in the Hanford Tank Farms (GJPO-HAN-1). The current calibration report for the logging tool utilized for logging borehole B8817 is Hanford Tank Farms Vadose Zone, Sixth Biannual Recalibration of Spectral Gamma-Ray Logging Systems Used for Baseline Characterization Measurements in the Hanford Tank Farms (GJO-HAN-26).

Casing corrections were applied to the data to compensate for the gamma-ray attenuation by the casing. The wall thickness of each casing string utilized in borehole B8817 was measured with a micrometer. The resulting values were applied to correct the data at the appropriate depth intervals. Additionally, constants determined for casing corrections during calibrations are determined for a single-cased borehole/well configuration. In wells where there are depth intervals where more than one casing is in place, the wall thicknesses are assumed to be additive to derive the proper correction. Although this assumption approximates the proper correction, it is known this correction could be slightly in error. The exact casing configuration and wall thicknesses used during data processing are discussed later in this report.

Water corrections were applied to the log data where appropriate. Attenuation of gamma rays from water can be significant and corrections to log data are derived for various borehole/well diameters during system calibrations. This correction is applied to the log data during data processing.

Repeat sections were conducted during some log surveys to document the logging system repeatability. Additionally, there are depth overlaps of $\log$ data where separate log runs were conducted. These overlaps are also useful to evaluate system repeatability. 
Neutron-Neutron Moisture Data

The neutron-neutron moisture data were acquired in spectral form and converted to gross counts for each $0.250-\mathrm{ft} \log$ interval. The counts for each log interval were converted to volume fraction moisture (volume water/volume total) using the calibration transform described in Radionuclide Logging System In-Situ Vadose Zone Moisture Measurement Calibration (WHC-SD-EN-TI-306, Rev. 1).

Descriptions of the accuracy and precision estimates for the neutron-neutron measurements are presented in the following discussions. Accuracy is the instrumentation response compared to the calibration model values. The precision is a function of the counting statistics and the instrument response. Both of these characterizations of the instrumentation utilized for this project are quantified and displayed.

\section{Accuracy}

The accuracy of the instrumentation as established by the Data Quality Objectives (DQO) for moisture logging instrumentation is to be determined by the comparison of calibrated instrument response to the assigned model values of moisture content as per PNNL assignment during construction PNL-10801, UC606, "Calibration Models for Measuring Moisture in Unsaturated Formations by Neutron Logging", Engleman, et al., October, 1995). The following table provides the results for the 8-in.-diameter casing calibration data that was collected on May 13, 1999 for the logging tool utilized for this project.

\begin{tabular}{|c|c|c|}
\hline $\begin{array}{l}\text { 8-in. casing model moisture } \\
\text { (volume fraction water in \%) }\end{array}$ & $\begin{array}{l}\text { Calibrated logging tool results } \\
\text { (volume fraction water in \%) }\end{array}$ & $\begin{array}{l}\text { Percent difference between model } \\
\text { value and instrument measurement }\end{array}$ \\
\hline 19.7 & 19.66 & -0.2 \\
\hline 11.9 & 11.79 & -0.9 \\
\hline 5.0 & 5.01 & +0.2 \\
\hline
\end{tabular}

The largest difference between the moisture model values and the logging tool measurements in the three Moisture Models is less than 1\% volume fraction of moisture. These observed deviations are within the limits of accuracy established by BHI for this project.

\section{Precision}

Precision is the ability of an instrument to reproduce a given measurement; therefore, the variance observed for a given repeat sequence is used to determine the system or instrument's precision. During model data collection, 10 samples or repeat measurements are acquired in each model. These data are then used to compute a root mean square or estimated observed standard deviation. These data are also used to compute a theoretical standard deviation based solely on counting statistics, and the comparison agreed within statistical limits of sample size.

The model data collected for the calibration demonstrated that the observed deviations for any model were the same as predicted by the counting statistics (i.e., no extraneous instrumentation error were introduced). Therefore it is possible to predict the statistical precision for any given logging condition. The total counts observed for each depth sample determine the statistical precision. Furthermore, because the countrate is a function of the observed moisture content, it is possible to derive the statistical precision as a function of moisture for either calibrated casing configuration.

The sample acquisition time per depth increment is multiplied by the projected countrate from the calibration coefficients. This results in the number of counts for each logging sample as a function of 
moisture content. Since the moisture log countrate follows a Poisson distribution, the standard deviation for each sample location is equal to the square root of the number of counts measured in the sample. For the given borehole logging, the highest logging speed was 1.0 feet per minute ( $\mathrm{ft} / \mathrm{min}$ ) with a sampling frequency of one sample per $0.25 \mathrm{ft}$. The resulting sampling time is therefore 15 seconds or more. The casing thickness correction for the 0.500 -in. wall thickness casing is 0.833 . The countrate to volume fraction of moisture content conversion is performed by the following equation:

$$
\mathrm{V}=\mathrm{FaC}^{\alpha},
$$

Where $\mathrm{V}$ is the instrument reading of volume fraction of moisture, $\mathrm{F}$ is the casing correction factor, $\mathrm{C}$ is the observed countrate, and the coefficients a and $\alpha$ are the calibration coefficients. For the logging tool utilized for this project, the calibration coefficients were determined to be:

$\mathrm{F}=1.2$

$\mathrm{a}=1.597 \mathrm{E}-05$

$\alpha=2.537$

The precision (or repeatability) of the neutron moisture measurements were determined for each of the measurements, and are indicated on the moisture plots as error bars showing the uncertainty at $+1-1$ sigma (67 percent confidence interval). The values of volumetric moisture content in borehole B8817 range from less than 1 percent to as high as 15 percent at a depth of $132 \mathrm{ft}$. The neutron countrate increased sharply at a depth of $200 \mathrm{ft}$ as groundwater was encountered. The calculated moisture values were not plotted at the bottom of the borehole since a higher scale would reduce the resolution of features for the rest of the moisture plot.

\section{Bulk Density Effects}

The assumed bulk density for the moisture $\log$ is the bulk density of the moisture calibration models and is 1.76 grams per cubic centimeter $(\mathrm{g} / \mathrm{cc})$.

Due to the lack of continuous measurements of bulk density over the interval logged bulk density corrections to the moisture data are not possible.

The document Radionuclide Logging System In-Situ Vadose Zone Moisture Measurement Calibration (WHC-SD-EN-TI-306, Rev. 1) provides a density correction curve in Figure 6 that is based on computer modeling. This data could be used to provide an estimate of how the density assumption affects accuracy but is not provided herein because accuracy and precision statements of recommendations made per the Geophysical Logging Master Agreement are for "performance under known conditions such as in calibration models."

\section{Casing Thickness Corrections}

A casing thickness correction for the 8-in.-diameter casing was applied to the moisture measurements in accordance with WHC-SD-EN-TI-306, Rev. 1. Since there was no direct calibration of the neutron moisture logging tool in 11 -in.-diameter casing, the region of borehole B8817 with 11-in.-diameter casing was not logged. 


\section{Log Plots}

Separate $\log$ plots show the man-made and naturally occurring radionuclides potassium $-40\left({ }^{40} \mathrm{~K}\right)$, uranium-238 $\left({ }^{238} \mathrm{U}\right)$, and thorium-232 $\left({ }^{232} \mathrm{Th}\right)$. The heading for each of the radionuclide $\log$ plots indicate the energy peak utilized to identify the specific radionuclide.

Various log plots show the concentrations of the individual radionculides, the total gamma count rate, and the volumetric moisture content. The total gamma count rate for each $0.5-\mathrm{ft}$ depth increment is a sum of the counts from the spectrum for the particular depth increment divided by the counting time. However, the total gamma countrate is not corrected for water or casing, so a direct relationship between concentration and countrate does not exist. For example, in a borehole that was logged in two separate $\log$ runs, one run within a single casing and the other within two casings, the KUT concentrations will approximately repeat in both configurations as a result of the casing corrections. However, the total gamma rate will be much lower in the two-casing configuration run when compared to the single-casing $\log$, because no casing correction is applied to the total gamma log data.

The KUT plots allow correlation of the log data with lithologic features, with intervals of man-made contamination, if it exists, and with moisture content. The statistical uncertainty for gamma-rays emitted from low-concentration radionuclides such as ${ }^{238} \mathrm{U}$ and ${ }^{232} \mathrm{Th}$ can be high for the 100 -s acquisition time utilized for logging and the plots of the concentration of these radionuclides show high levels of uncertainty. This uncertainty is shown on the KUT plots by scattering of the data and large error bars. Nevertheless, these data are useful for correlation with geologic data or other borehole logs, e.g. moisture content, contamination intervals, etc.

Error bars shown on the log plots include the error associated with calibration measurements, and error associated with the casing and water corrections as well as counting error. Therefore, increased measurement error as shown on the log plots is the result of data collection that occurred in regions of boreholes containing water, containing casings of greater wall thickness (compared to conventional steel casing), and regions of boreholes where overlapping casings occur. This error is enhanced with increasing radionuclide concentrations.

The minimum detection limit (MDL) of a radionuclide represents the lowest concentration at which positive identification of a gamma-ray peak for that radionclide is statistically defensible. The spectral analysis program calculates the MDL for a particular peak on the basis of a statistical comparison with the background energy in the vicinity of the peak. An MDL for a particular radionuclide increases when additional gamma attenuation is introduced as a result of multiple casings or water in the borehole. Additionally, an MDL may increase with increasing radionuclide concentrations. The MDL's for the radionuclides are indicated on the plots by open circles.

The plots of the volumetric moisture content measured in borehole B8817 is provided with error bars for approximately every fifth moisture measurement. More frequent presentation of measurement error (error bars) obscures features of the log plot. The error bars represent the 68-persent confidence level ( + 1 standard deviation), which was determined by converting the square root of the counts in each interval to equivalent moisture content. The definition of "Detection Limit" or threshold limit is defined as the minimum concentration or level at which a contaminant or natural element can be positively be identified. Since moisture can be measured to zero volume fraction, threshold limit is not applicable to moisture measurements and is not indicated on the log plots. 


\section{Results}

Borehole B8817 is located at the 216-S-10 Pond southwest of the 200 West Area. The borehole was drilled in November and December 1999 to a depth of $240.7 \mathrm{ft}$. A nominal 11-in.-diameter casing was used to advance the borehole from ground surface to a depth of $36.3 \mathrm{ft}$, and an 8-in.-diameter casing was used to drill to a depth of $240 \mathrm{ft}$. A short section of the borehole between depths of 240.0 and 240.7 was uncased. Casing wall thickness was measured in the field using a micrometer; the wall thickness of each of the casings is $0.5 \mathrm{in}$. At the time of logging, both casing strings were set approximately flush with ground surface. The depth to groundwater on the day the borehole was logged was measured at $200.49 \mathrm{ft}$.

Spectral gamma logging in borehole B8817 was conducted in four $\log$ runs. Log runs 1 and 2 were conducted on December 9, 1999 inside the 8-in.-diameter casing. Log run 1 covered the depth interval from 240.5 to $119.0 \mathrm{ft}$. Log run 2 was a repeat survey between depths of 205.0 and $193.0 \mathrm{ft}$. Log runs 3 and 4 were conducted on December 10, 1999 inside the 11-in.- and 8-in.-diameter casings. Log run 3 covered the interval between ground surface and a depth of $125.0 \mathrm{ft}$. Log run 4 was a repeat section between depths of 34.0 and $46.0 \mathrm{ft}$. In addition, a short overlap of data occurs between depths of 119.0 and $125.0 \mathrm{ft}$ where the survey intervals of $\log$ runs 1 and 3 overlapped.

A generalized casing correction function was used to determine casing correction factors. Log runs 1 and 2 were analyzed using a correction for casing with a wall thickness of 0.5 in. Log runs 3 and 4 were analyzed using a correction for 0.5 in.-thick casing in the 8-in.-diameter section of the surveys (between depths of 36.3 and $125.0 \mathrm{ft}$ ); a correction for 1.0-in-thick casing was applied to the data acquired in the section of the borehole where both the 11-in.- and 8-in.-diameter casings were present (ground surface to a depth of $36.3 \mathrm{ft}$ ). With more than one casing in place, the wall thickness is assumed to be an additive of both casings to derive the proper correction factor. These corrections are applied to the raw data that are recorded in counts per second to adjust the countrates for the attenuation that is caused by the presence of the casing.

Water corrections were applied to the log data collected between depths of 200.49 (the measurement on the day of logging) and $240.5 \mathrm{ft}$. Attenuation of gamma rays by water can be significant, which is reflected by the sporadic uranium- $238\left({ }^{238} \mathrm{U}\right)$ concentrations below a depth of $200.5 \mathrm{ft}$.

Separate log plots show the man-made and naturally occurring radionclides potassium- $40\left({ }^{40} \mathrm{~K}\right)$, uranium$238\left({ }^{238} \mathrm{U}\right)$, and thorium-232 $\left({ }^{232} \mathrm{Th}\right)(\mathrm{KUT})$; there were no man-made radionuclides detected in borehole B8817. For calculating ${ }^{238} \mathrm{U}$ concentrations between ground surface and a depth of $36.5 \mathrm{ft}$, the 1764.51 $\mathrm{keV}$ bismuth-214 $\left({ }^{214} \mathrm{Bi}\right)$ gamma ray was utilized instead of the routinely used $609.32-\mathrm{keV}{ }^{214} \mathrm{Bi}$ gamma ray. For many of the spectra in this depth region of the borehole, the $609.32-\mathrm{keV} 214 \mathrm{Bi}$ peak was not detected because of the attenuation resulting from the 1.0-in.-thick double casing. Although the yield (intensity) is lower for the $1764.51-\mathrm{keV}$ peak, the higher energy level results in less attenuation by the double casing. The headings of the plots indicate the specific gamma peak that is used to determine the concentrations of the radionuclides. The error bars on the plots represent counting errors as well as errors associated with casing corrections, deadtime corrections, and the calibration function. The error bars represent the statistical uncertainties for the measurements as 95-percent (2-sigma) confidence intervals. Open circles on the plots indicate the minimum detectable level (MDL) of the particular radionuclide. The MDL for a specific radionuclide represents the lowest concentration at which positive identification of the gamma-ray peak is statistically defensible. MDLs generally increase in areas of higher gamma activity, and where additional gamma-ray attenuation occurs as a result of the presence of water or casing with greater wall thickness.

The standard combination log plot includes both the man-made and naturally occurring radionuclides, as well as a plot of the total gamma-ray activity as derived form the gamma spectra. The total gamma count 
rate for each 0.5 - $\mathrm{ft}$ depth interval is a sum of the counts from the spectra divided by the counting time. The combination plots are composites of data acquired in log runs 1 and 3.

Repeat $\log$ plots compare the repeat surveys from runs 2 and 4 with $\log$ runs 1 and 3 , respectively. Repeat surveys were conducted to document the logging system's repeatability. These plots show good repeatability for the calculated radionuclide concentrations and depth control.

The plot of the naturally occurring radionculides shows several distinct concentration changes at depths of $50 \mathrm{ft}, 102 \mathrm{ft}, 133 \mathrm{ft}, 195$, and near the bottom of the borehole at a depth of $237 \mathrm{ft}$. Some of these changes correlate to lithologic features recorded on the borehole geologic log and with the moisture log data. Error bars representing the 2-sigma uncertainty in the measurements increase above a depth of $36 \mathrm{ft}$ and below a depth of $200 \mathrm{ft}$ as a result of additional error introduced by the corrections for double casing and the presence of water.

The combination plot is intended to show both the man-made and naturally occurring radionuclides (KUT), in addition to the total gamma countrate. Because the total gamma countrate is not corrected for casing or water, the total gamma decreases above a depth of $26 \mathrm{ft}$ and below a depth of $200 \mathrm{ft}$ where the double casing and water are present, respectively. The changes observed in the KUT concentrations are reflected in the profile of the gross gamma countrate in particular at the depths mentioned previously.

Moisture logging was conducted in borehole B8817 in the region of the borehole with 8-in.-diameter casing, below the $11-$ in. diameter casing $(36.3 \mathrm{ft})$ and above the groundwater level $(200.5 \mathrm{ft})$. The logging tool utilized for moisture logging for this project is not calibrated for use in 11-in.-diameter casing. Vigorous calibration has been performed with this logging tool in 6-in.- and 8-in.-diameter casings. A short interval of the borehole between depths of 34 and $36.3 \mathrm{ft}$ was logged to identify the bottom of the 11-in.-diameter casing, and a repeat survey was conducted between depths of 100 and 121.5 $\mathrm{ft}$ to verify system performance.

The volumetric moisture content ranges between 2 and 15 percent throughout most of the logged interval. The highest moisture content value of almost 15 percent was measured at a depth of $134 \mathrm{ft}$. Error bars representing one standard deviation are presented for every fifth moisture measurement for clarity of data presentation.

A short interval was logged in both the 11 -in.- and 8-in.-diameter casings ( 34 to $36.3 \mathrm{ft}$ ) to identify the bottom of the 11 -in.-diameter casing. A decrease in the moisture content values should have been noticeable due to the attenuation resulting from the presence of both casings; however, a peak of elevated moisture content was observed at a depth of $35 \mathrm{ft}$. The reason for these elevated moisture values is unknown since the sediments at this depth are described as slightly moist.

The most obvious features on the moisture content plot are the fluctuating moisture values between depths of 90 and about $130 \mathrm{ft}$ where several narrow peaks occur. In this region of the borehole, the moisture content values range between 2 and 14 percent. The geologic log for this borehole indicates the sediments in this region of the borehole range from mostly sand ( 95 percent) with 1 to 2 in.-thick beds of silt, to slightly silty sand, sandy silt, and silty sand. The peaks of higher moisture content are most likely indicating finer grained materials that retain moisture. There is a very distinctive increase in the KUT concentrations in this region of the borehole.

The highest volumetric moisture content value of almost 15 percent occurs between depths of 134 and $136 \mathrm{ft}$. A caliche zone is identified on the geologic $\log$ between depths of 134 and $139 \mathrm{ft}$. Sediments of elevated moisture appear to lie on top of this caliche zone. Below the caliche zone to the bottom of the borehole, the sediments are mostly silty sandy gravel, sand, gravelly sand, and sandy gravel. There are no 
definitive lithologic features within this region of the borehole with which to correlate the features on the moisture plot, e.g. the peaks of elevated moisture content at depths of 151 and $176 \mathrm{ft}$, and the region of elevated moisture content between depths of 154 and $169 \mathrm{ft}$. The moisture content values increase at thebottom of the logged interval as groundwater is encountered.

\section{Data}

One compact disk is provided with this report and it contains the following data files:

B8817.JNB SigmaPlot version 4.0 format of spectral gamma plots for data acquired in borehole B8817

B8817M.JNB SigmaPlot version 4.0 format of neutron-neutron moisture and moisture-total gamma plots for data acquired in borehole B8817

B8817NNLH.DOC Word 97 format of the neutron-neutron moisture log header for borehole B8817

B8817NNRpt.DOC Word 97 format of the neutron-neutron moisture summary report for borehole B8817

B8817LH.PDF Adobe Acrobat PDF format of the spectral gamma log header for borehole B8817

B8817ANA.XLS Microsoft Excel 97 spreadsheet of analysis (spectral) data for borehole B8817

B8817MANA.XLS Microsoft Excel 97 spreadsheet of analysis (moisture) data for borehole B8817

200-CS-1RCRARPT.DOC Word 97 format of the report text 


\title{
Neutron-Neutron Moisture Borehole Survey
}

\author{
Log Header
}

Project: RCRA Drilling 1999

Well: 299-W26-13

Borehole Information

Well: \# 299-W26-13

Elevation Reference: n/a

Depth Reference: Ground surface

Casing Diameter: $\underline{11.75}$ in

Casing Diameter: 8.625 in
Water Depth: $200.49 \mathrm{ft}$

Elevation: $\underline{\mathbf{n}} \mathbf{a}$

Casing Stickup: $11.75-$ in. $-0 \mathrm{ft}: 8.625 \mathrm{in} .-0.1 \mathrm{ft}$

Depth Interval: 0 to $36.3 .0 \mathrm{ft} \quad$ Thickness: $0.5 \mathrm{in}$.

Depth Interval: $\underline{0 \text { to } 240 \mathrm{ft}}$ Thickness: $\underline{0.5}$ in.

\section{Logging Information}

\author{
Log Type: \\ Company: \\ Date: \\ Logging Engineer(s): \\ Instrument Series: \\ Logging Unit: \\ Depth Interval: \\ Instrument Calibration Date \\ Calibration Report:
}

\author{
Neutron-neutron moisture \\ Waste Management Technical Services \\ December 10, 1999. \\ Steven Kos \\ RLSM00.0 \\ RLS-1 \\ 34 to $121.5 \mathrm{ft}$ \\ 100 to $201.1 \mathrm{ft}$ \\ Prefix A686 \\ A687 \\ May 13, 1999 \\ WHC-SD-EN-TI-306, Rev.0
}

\section{Analysis Information}

$\begin{array}{ll}\text { Company: } & \text { Waste Management Technical Services } \\ \text { Analyst: } & \text { Steven Kos } \\ \text { Date: } & \text { January 15, 2000 } \\ \text { Depth Reference: } & \text { Ground Surface }\end{array}$

Notes Measurements were acquired at 0.250 - $\mathrm{ft}$ depth increments at a logging speed of $1.0 \mathrm{ft}$ per minute. The borehole was logged in one log survey in the 8 -in.-diameter casing. A short interval was logged in the 11 -in.-diameter and 8 -in.-diameter casings between depths of 34 and $36.3 \mathrm{ft}$. The survey was terminated when the logging tool reached groundwater at a depth of $201.1 \mathrm{ft}$. A repeat survey was conducted between depths of 100 and $121.5 \mathrm{ft}$. 


\section{Neutron-Neutron Moisture Survey}

Waste Management Technical Services

Project: RCRA Drilling 1999 Log Date : December 10, 1999

Borehole: 299-W26-13 Depth Datum: Ground Level

Moisture Vol. \%

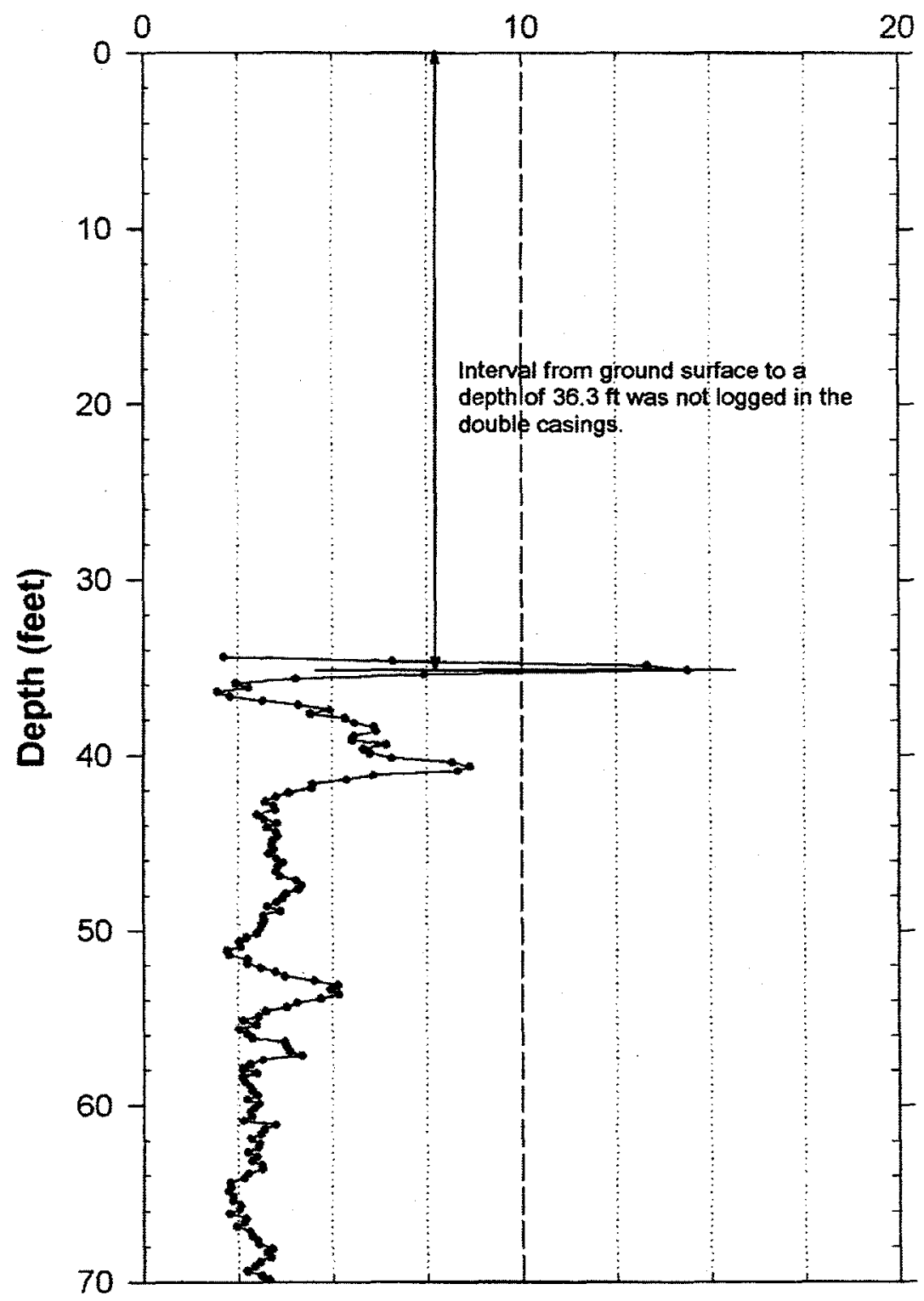




\section{Neutron-Neutron Moisture Survey \\ Waste Management Technical Services}

Project: RCRA Drilling 1999

Borehole: 299-W26-13
Log Date :December 10, 1999

Depth Datum: Ground Level

Moisture Vol. \%

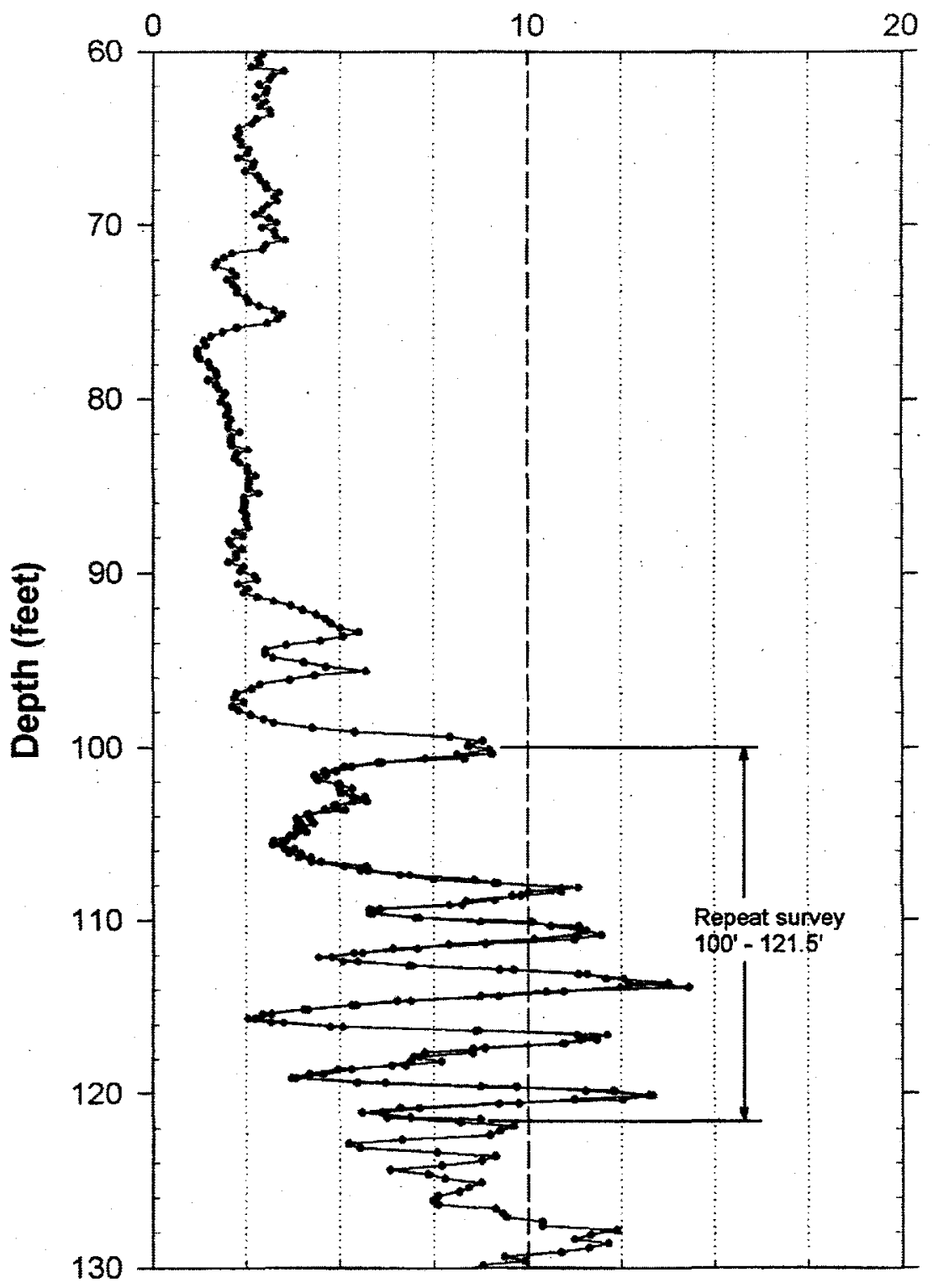




\section{Neutron-Neutron Moisture Survey}

Waste Management Technical Services

Project: RCRA Drilling 1999 Log Date: December 10, 1999 Borehole: 299-W26-13 Depth Datum: Ground Level

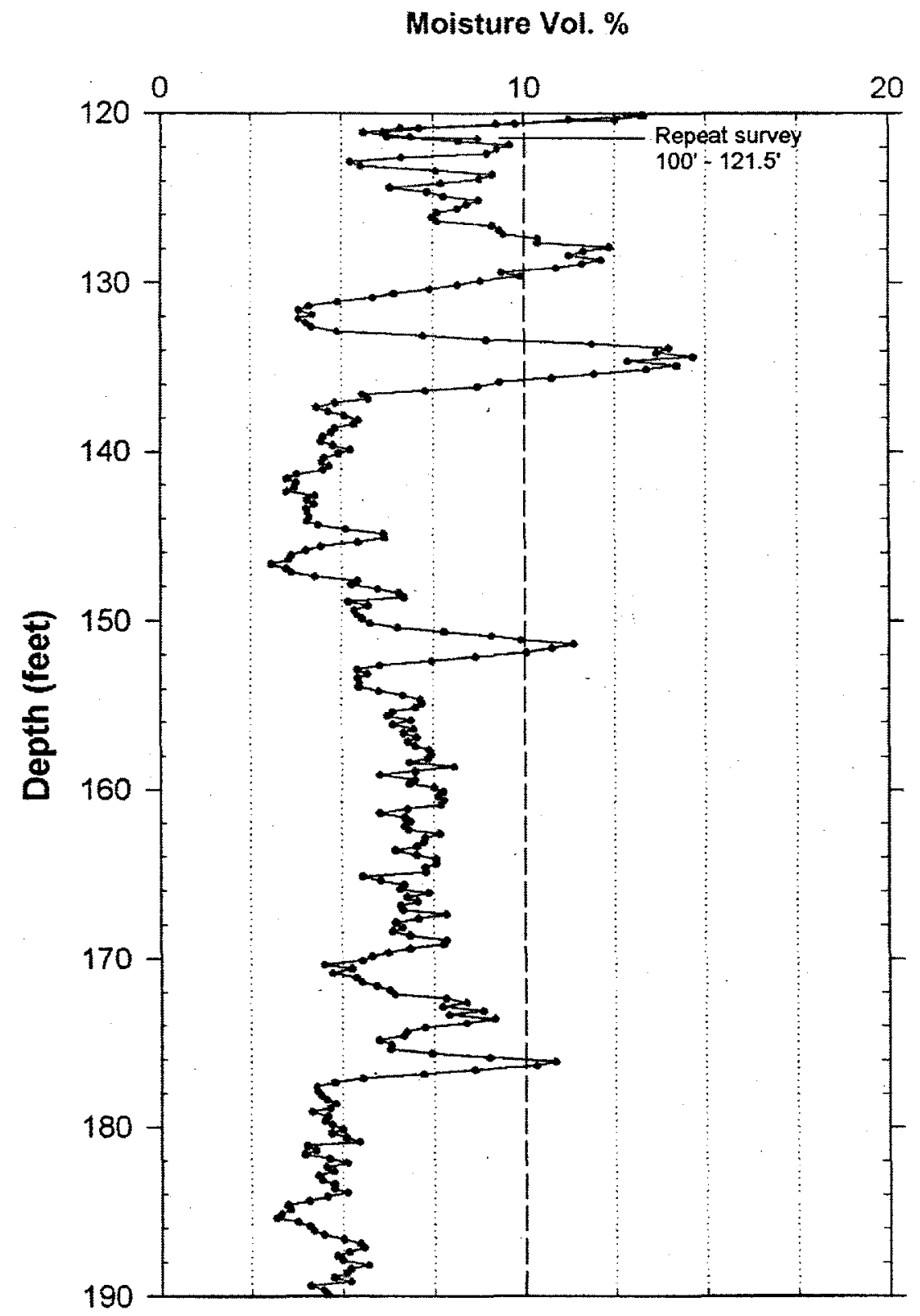




\section{Neutron-Neutron Moisture Survey \\ Waste Management Technical Services}

Project: RCRA Drilling 1999 Log Date: December 10,1999 Borehole: 299-W26-13 Depth Datum: Ground Level

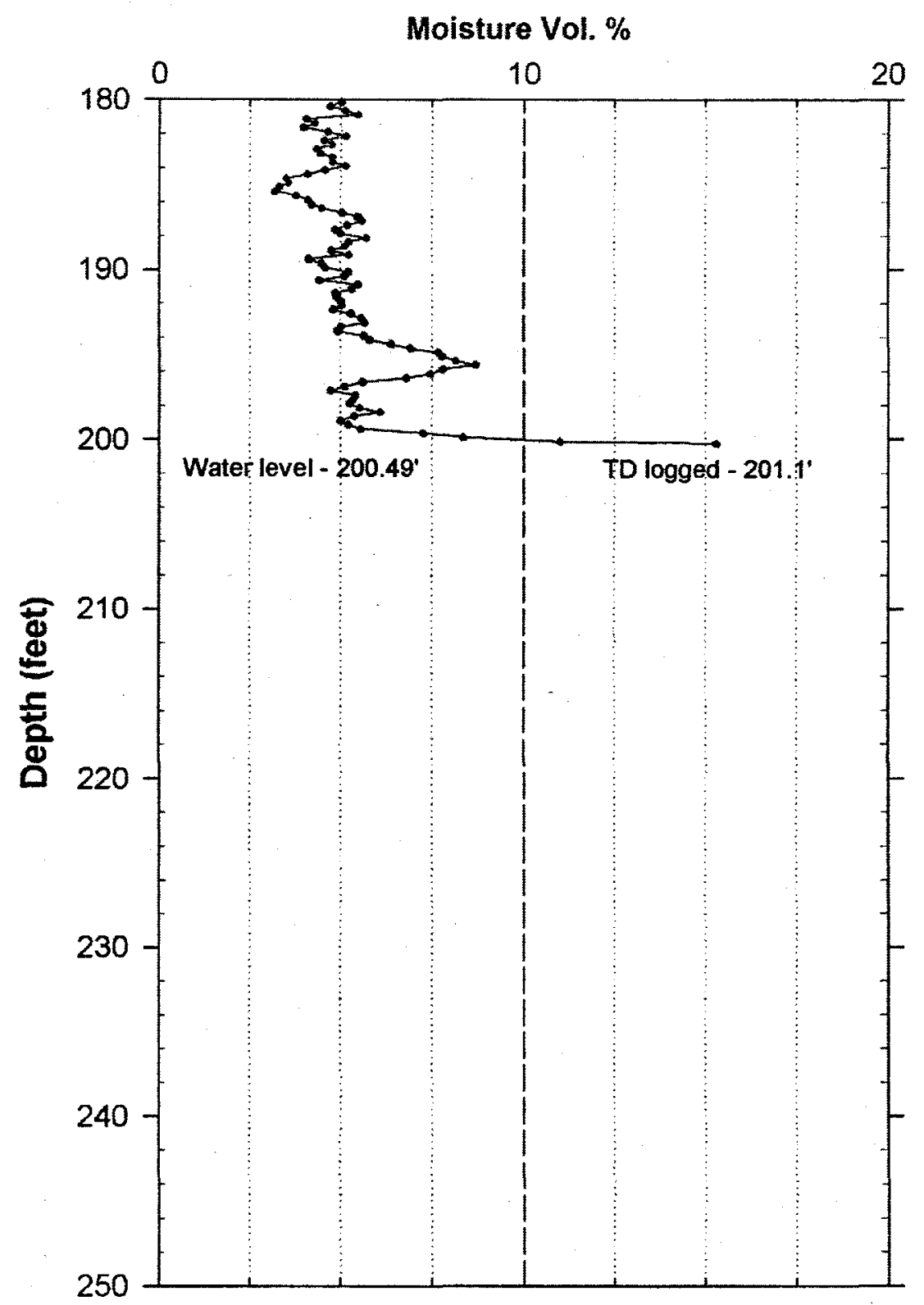




\section{Neutron-Neutron Moisture Borehole Survey \\ Waste Management Technical Services}

\section{Log Analysis Summary Report}

Project: 200-CS-1/RCRA

Log Type: Neutron-neutron moisture
Well: 299-W26-13

Log Date: December 10, 1999

\section{General Notes:}

All log data were collected relative to ground surface.

Logging was conducted in one $\log$ run in the 8-in.-diameter casing. Data were not acquired in the 11-in.diameter casing between ground surface and a depth of $36.3 \mathrm{ft}$ since the logging tool utilized for this project is not calibrated for this size casing.

Measurement uncertainties of one standard deviation (1 sigma) were calculated for all moisture measurements; for clarity in presentation of the log data, error bars were plotted for every fifth data point.

\section{System Performance Verify:}

The pre- and post- survey calibrations indicated the logging system was performing within specifications outlined in the logging procedures.

\section{Repeat Interval:}

A repeat survey was performed between depths of 100 and $121.5 \mathrm{ft}$. The results of the repeat surveys indicate the logging system was operating within specifications.

\section{Environmental Corrections:}

The countrates measured with the neutron-neutron logging tool were corrected for the appropriate casing thickness. 


\section{Distribution}

No. of

Copies

OFFSITE

L. Seelatsee

Wanapum Band

Grant County P.U.D.

30 "C" Street S.W.

P. O. Box 878

Ephrata, WA 98823

P. Sobotta

Nez Perce Tribe

Environmental Restoration/Waste

Management

P.O. Box 365

Lapwai, ID 83540-0365

Confederated Tribes of the Yakama Nation

Environmental Restoration/Waste

Management

2808 Main Street

Union Gap, WA 98903

ATTN: R. Jim

\section{ONSITE}

2 DOE Richland Operations Office
No. of

Copies

4 CH2M Hill Hanford, Inc.

J. V. Borghese

H9-03

C. S. Cearlock (3)

H9-03

4 Washington State Department of Ecology

D. L. Becker-Khaleel

B5-18

D. Goswami

B5-18

A. D. Huckaby

B5-18

S. Leja

B5-18

U.S. Environmental Protection Agency

D. A. Faulk

B5-01

15 Pacific Northwest National Laboratory

D. G. Horton (3)

K6-81

S. P. Luttrell

K6-96

W. J. Martin

K6-81

B. A. Williams (3)

K6-81

Information Release Office (7)
M.J. Furman (2)

2 CH2M Hill Group
A. J. Knepp
H0-22
D. C. Weekes
H9-02

A5-13

Distr.1 